\title{
TRANSFINITE DIAMETER ON COMPLEX ALGEBRAIC VARIETIES
}

\author{
DAVID A. COX AND SIONE MA'U
}

\begin{abstract}
We use methods from computational algebraic geometry to study Chebyshev constants and the transfinite diameter of a pure $m$-dimensional affine algebraic variety in $\mathbb{C}^{n}(m \leq n)$. The main result is a generalization of Zaharjuta's integral formula for the Fekete-Leja transfinite diameter.
\end{abstract}

\section{INTRODUCTION}

This paper studies a notion of transfinite diameter on a pure $m$-dimensional algebraic subvariety of $\mathbb{C}^{n}, 1 \leq m \leq n$. This is a natural generalization of the FeketeLeja transfinite diameter in $\mathbb{C}^{n}$, which is an important quantity in pluripotential theory and polynomial approximation. In the study of the Fekete-Leja transfinite diameter in $\mathbb{C}^{n}(n>1)$, an important paper is that of Zaharjuta 11. Given a compact set $K \subseteq \mathbb{C}^{n}$, Zaharjuta showed that its Fekete-Leja transfinite diameter, denoted $d(K)$, was given by a well-defined limiting process analogous to the onedimensional case. The main result of 11 is an integral formula that realizes $d(K)$ as a "geometric average" of so-called directional Chebyshev constants associated to $K$; these constants measure (in an asymptotic sense) the minimum size on $K$ of polynomials with prescribed leading terms.

Further developments and generalizations make use of the essential techniques in 11. In 7 the notion of homogeneous transfinite diameter was studied and a Zaharjuta-type formula proved. In [8, and later in [9, Lau, Rumely and Varley developed Zaharjuta's techniques in the setting of arithmetic geometry to study the notion of sectional capacity. More recently, Bloom and Levenberg studied a notion of weighted transfinite diameter in $\mathbb{C}^{n}([3,4])$.

In 1 a notion of transfinite diameter was defined and studied on an algebraic curve $V \subseteq \mathbb{C}^{n}$. It was shown that Zaharjuta's arguments, which exploit standard algebraic properties of polynomials, may be adapted to handle algebraic computations in the coordinate ring of $V$. Well-developed methods exist to carry out such computations, using Groebner bases. In this paper we will apply these methods to higher dimensional algebraic varieties.

We should mention here that the notion of transfinite diameter on algebraic varieties may be studied as a by-product of Berman and Boucksom's general theory of Monge-Ampère energy on compact complex manifolds [2]. Their methods are quite different to those of this paper.

2010 Mathematics Subject Classification. 32U20; 14Q15.

Key words and phrases. Chebyshev constant, transfinite diameter, Vandermonde determinant, affine variety, Noether normalization, monomial order. 
Before we describe the contents of the paper more specifically, we briefly recall the definition of the Fekete-Leja transfinite diameter.

Let $\left\{z^{\alpha_{j}}\right\}_{j=1}^{\infty}$ be the monomials in $n$ variables listed according to a graded order (i.e., $\left|\alpha_{j}\right| \leq\left|\alpha_{k}\right|$ whenever $j<k$ ). Here we are using standard multi-index notation: if $\alpha_{j}=\left(\alpha_{j 1}, \ldots, \alpha_{j n}\right) \subseteq \mathbb{Z}_{\geq 0}^{n}$, then $z^{\alpha_{j}}=z_{1}^{\alpha_{j 1}} z_{2}^{\alpha_{j 2}} \cdots z_{n}^{\alpha_{j n}}$ and $\left|\alpha_{j}\right|=\alpha_{j 1}+\cdots+\alpha_{j n}$ denotes the total degree. Write $\mathbf{e}_{j}=z^{\alpha_{j}}$; so for $a=\left(a_{1}, \ldots, a_{n}\right) \in \mathbb{C}^{n}$ we have $\mathbf{e}_{j}(a)=a_{1}^{\alpha_{j 1}} \cdots a_{n}^{\alpha_{j n}}$. Given a positive integer $M$ and points $\left\{\zeta_{1}, \ldots, \zeta_{M}\right\} \subseteq \mathbb{C}^{n}$, the $M \times M$ determinant

$$
\operatorname{Van}\left(\zeta_{1}, \ldots, \zeta_{M}\right)=\operatorname{det}\left(\mathbf{e}_{j}\left(\zeta_{i}\right)\right)_{i, j=1}^{M}=\operatorname{det}\left(\begin{array}{cccc}
1 & 1 & \cdots & 1 \\
\mathbf{e}_{2}\left(\zeta_{1}\right) & \mathbf{e}_{2}\left(\zeta_{2}\right) & \cdots & \mathbf{e}_{2}\left(\zeta_{M}\right) \\
\vdots & \vdots & \ddots & \vdots \\
\mathbf{e}_{M}\left(\zeta_{1}\right) & \mathbf{e}_{M}\left(\zeta_{2}\right) & \cdots & \mathbf{e}_{M}\left(\zeta_{M}\right)
\end{array}\right)
$$

is called a Vandermonde determinant of order $M$. (Note that $\mathbf{e}_{1}=1$.)

Let $K \subseteq \mathbb{C}^{n}$ be compact and $s$ a positive integer. Let $m_{s}$ be the number of monomials of degree at most $s$ in $n$ variables, and let $l_{s}=\sum_{j=1}^{m_{s}}\left|\alpha_{j}\right|$ be the sum of the degrees. Define the $s$-th order diameter of $K$ by

$$
d_{s}(K):=\sup \left\{\left|\operatorname{Van}\left(\zeta_{1}, \ldots, \zeta_{m_{s}}\right)\right|^{\frac{1}{l_{s}}}:\left\{\zeta_{1}, \ldots, \zeta_{m_{s}}\right\} \subseteq K\right\}
$$

The Fekete-Leja transfinite diameter of $K$ is defined as $d(K):=\limsup _{s \rightarrow \infty} d_{s}(K)$.

In this paper, we construct a basis $\mathcal{C}$ of polynomials for the coordinate ring $\mathbb{C}[V]$ of a pure $m$-dimensional algebraic variety $V \subseteq \mathbb{C}^{n}(1 \leq m \leq n)$ of degree $d$, as long as the ring satisfies certain algebraic conditions (see (3.1)). Write $\mathcal{C}=\left\{\mathbf{e}_{j}\right\}_{j=1}^{\infty}$ for this basis which we assume is listed in a graded ordering: $\operatorname{deg}\left(\mathbf{e}_{j}\right) \leq \operatorname{deg}\left(\mathbf{e}_{k}\right)$ if $j<k$. We define $\operatorname{Van}_{\mathcal{C}}\left(\zeta_{1}, \ldots, \zeta_{M}\right)$ to be the Vandermonde determinant with respect to $\mathcal{C}$ using the formula (1.1).

Define $m_{s}=m_{s}(V)$ to be the number of elements of $\mathcal{C}$ of degree at most $s$, and let $l_{s}=l_{s}(V)=\sum_{j=1}^{m_{s}} \operatorname{deg}\left(\mathbf{e}_{j}\right)$ be the sum of the degrees. The $s$-th order diameter of a compact set $K \subseteq V$ is defined as in (1.2) with $\operatorname{Van}_{\mathcal{C}}(\cdot)$ replacing $\operatorname{Van}(\cdot)$ on the right-hand side. Our main theorem (Theorem 6.2) says the following.

Theorem. The limit $d(K):=\lim _{s \rightarrow \infty} d_{s}(K)$ exists and

$$
d(K)=\left(\prod_{j=1}^{d} T\left(K, \lambda_{j}\right)\right)^{\frac{1}{d}} .
$$

Following Zaharjuta's terminology, the quantities $T\left(K, \lambda_{j}\right)$ on the right-hand side are called principal Chebyshev constants and are defined in Section 5 as integral averages of so-called directional Chebyshev constants. Here $d$ is the degree of $V$ and the $\lambda_{j}$ 's are the $d$ points of intersection of the projective closure of $V$ in $\mathbb{P}^{n}$ with a certain subspace of the hyperplane at infinity. When $V$ is a curve the above result is in [1] When $\operatorname{deg}(V)=1$ then there is only one principal Chebyshev constant, and one recovers Zaharjuta's formula, up to a normalization.

*The principal Chebyshev constants in this paper are called directional Chebyshev constants in [1] for a one-dimensional curve, the $\lambda_{j}$ 's may be interpreted as the directions of its linear asymptotes. 
In Section 2 we give some of the background needed for subsequent sections, including Noether normalization, the grevlex monomial ordering, normal forms and Hilbert functions.

In Section 3 we construct a basis (denoted by $\mathcal{C}$ ) of polynomials on the variety. The basis $\mathcal{C}$ consists of $d$ groups of polynomials associated to the Noether normalization (elements of the form $(* *)$, see Proposition 3.9), together with a "smaller" collection of monomials (elements of the form $(*)$ ). When $V$ is a hypersurface, the basis $\mathcal{C}$ can be computed rather explicitly.

Section 4 is a general study of weakly submultiplicative functions. In 3 it was observed that Zaharjuta's computations with polynomials can be reformulated abstractly as properties of submultiplicative functions. We verify here that the relevant calculations go through with small modifications under slightly weaker conditions.

In Section 5, directional and principal Chebyshev constants are defined and studied. The main point is to construct weakly submultiplicative functions using computational properties of the basis $\mathcal{C}$ (Corollary [5.4). The results of Sections 3 and 4 can then be applied to this setting.

In Section 6 we prove the main theorem relating transfinite diameter to Chebyshev constants. The standard argument, based on estimating ratios of Vandermonde determinants with directional Chebyshev constants, goes through in its entirety.

In Section 7, we show in Theorem 7.2 that the transfinite diameter may be computed using the standard basis of monomials on the variety (i.e., those monomials that give normal forms). This uses the fact that, up to a geometric factor in some finite set - the collection of $\mathbf{v}_{i}$ 's in Proposition 3.9 each polynomial in the basis $\mathcal{C}$ is a monomial.

In the appendix we compare our method to that of Rumely, Lau and Varley 9], whose so-called monic basis is constructed by generating basis elements multiplicatively from a finite collection of polynomials with prescribed behaviour. We compare both methods concretely in the case of the sphere in $\mathbb{C}^{3}$.

\section{BACKGROUND MATERIAL}

We begin with Noether normalization. Consider an ideal $I \subseteq \mathbb{C}\left[z_{1}, \ldots, z_{n}\right]$ with the following properties:

(1) $\mathbb{C}\left[z_{1}, \ldots, z_{m}\right] \cap I=\{0\}$; and

(2) For each $i=m+1, \ldots, n$ there exists a $g_{i} \in I$ which can be written in the form

$$
g_{i}=z_{i}^{d_{i}}+\sum_{j=0}^{d_{i}-1} h_{i j}\left(z_{1}, \ldots, z_{i-1}\right) z_{i}^{j}, \quad \text { with } \operatorname{deg}\left(h_{i j}\right)+j \leq d_{i} \text { for all } i \text {. }
$$

Property (10) is equivalent to saying that the map $\mathbb{C}\left[z_{1}, \ldots, z_{m}\right] \rightarrow \mathbb{C}\left[z_{1}, \ldots, z_{n}\right] / I$, induced by the inclusion into $\mathbb{C}\left[z_{1}, \ldots, z_{n}\right]$, is injective, and property (2) implies that the quotient is finite over $\mathbb{C}\left[z_{1}, \ldots, z_{m}\right]$. The Noether normalization theorem says that one can always make a change of variables so that the above properties hold. We state a specialized version of this theorem (cf. 10, Theorem 3.4.1).

Theorem 2.1 (Noether Normalization). Let $J \subseteq \mathbb{C}\left[x_{1}, \ldots, x_{n}\right]$ be an ideal. Then there is a positive integer $m \leq n$ and a complex linear change of coordinates $z=$ $T(x), z_{i}=\sum_{j=1}^{n} T_{i j} x_{j}$, such that the following properties hold (write $I=T(J)$ ): 
(1) The map $\mathbb{C}\left[z_{1}, \ldots, z_{m}\right] \rightarrow \mathbb{C}\left[z_{1}, \ldots, z_{n}\right] / I$ induced by inclusion is injective, and exhibits $\mathbb{C}\left[z_{1}, \ldots, z_{n}\right] / I$ as a finite $\mathbb{C}$-algebra over $\mathbb{C}\left[z_{1}, \ldots, z_{m}\right]$.

(2) For $i=m+1, \ldots, n$, we can find polynomials $g_{i} \in I$ that satisfy (2.1).

When property (1) of the theorem holds, we write $\mathbb{C}\left[z_{1}, \ldots, z_{m}\right] \subseteq \mathbb{C}\left[z_{1}, \ldots, z_{n}\right] / I$. This inclusion is called a Noether normalization. All Noether normalizations used in this paper will be assumed to satisfy the additional condition (2) of the theorem since the degree condition in (2.1) will be important.

The grevlex ordering, which we will denote here by $<_{g r}$, is the ordering defined on $\mathbb{Z}_{>0}^{n}$ by $\alpha<_{g r} \beta$ if:

(1) $|\alpha|<|\beta|$; or,

(2) $|\alpha|=|\beta|$, and for some $i \in\{1, \ldots, n\}$ we have $\alpha_{i}<\beta_{i}$ and $\alpha_{j}=\beta_{j}, \forall j<i$.

Define grevlex on monomials by putting $z^{\alpha}<_{g r} z^{\beta}$ if $\alpha<_{g r} \beta$. More precisely, this gives the grevlex ordering with $z_{1}<_{g r} z_{2}<_{g r} \cdots<_{g r} z_{n}$. Note that $|\alpha|<|\beta|$ implies $z^{\alpha}<_{g r} z^{\beta}$. A monomial ordering that satisfies this property is called a graded ordering.

Denote by $\operatorname{LT}(p)$ the leading term of a polynomial with respect to grevlex, and for an ideal $I$ put $\operatorname{LT}(I):=\{\operatorname{LT}(p): p \in I\}$. It is well-known that for each element of $\mathbb{C}\left[z_{1}, \ldots, z_{n}\right] / I$ there is a unique polynomial representative, the normal form (with respect to grevlex), which contains no monomials in the ideal $\langle\operatorname{LT}(I)\rangle$. If an element of $\mathbb{C}\left[z_{1}, \ldots, z_{n}\right] / I$ contains the polynomial $p$, then the normal form $r$ may computed in practice as the remainder on dividing $p$ by a Groebner basis of $I$ (cf. [5], §5.3).

Write $\mathbb{C}[z]_{I}=\mathbb{C}\left[z_{1}, \ldots, z_{n}\right]_{I}$ for the collection of normal forms of elements of $\mathbb{C}[z] / I=\mathbb{C}\left[z_{1}, \ldots, z_{n}\right] / I$. As a vector space, $\mathbb{C}[z]_{I}$ has a basis consisting of all monomials $z^{\gamma} \notin\langle\operatorname{LT}(I)\rangle$. We can give $\mathbb{C}[z]_{I}$ the structure of an algebra over $\mathbb{C}$ with multiplication operation given by

$$
\left(r_{1}, r_{2}\right) \longmapsto \text { "the normal form of } r_{1} r_{2} " .
$$

We will usually denote this by $r_{1} r_{2}$, though we will write $r_{1} * r_{2}$ when we want to emphasize that this is the normal form of the ordinary product. Note that $\mathbb{C}[z]_{I}$ and $\mathbb{C}[z] / I$ are isomorphic as $\mathbb{C}$-algebras, where the isomorphism is given by identifying normal forms with their polynomial classes.

Hilbert functions play an important role in some of our proofs. We begin with $\mathbb{C}[z]_{\leq s}=\mathbb{C}\left[z_{1}, \ldots, z_{n}\right]_{\leq s}$, which consists of polynomials of degree $\leq s$. Recall that

$$
\operatorname{dim} \mathbb{C}\left[z_{1}, \ldots, z_{n}\right]_{\leq s}=\left(\begin{array}{c}
s+n \\
n
\end{array}\right)=\frac{(s+n) \cdots(s+1)}{n !}=\frac{1}{n !} s^{n}+O\left(s^{n-1}\right) .
$$

Then $(\mathbb{C}[z] / I)_{\leq s}$ consists of all classes represented by a polynomial of degree $\leq s$. The dimension $\operatorname{dim}(\mathbb{C}[z] / I)_{\leq s}$ gives the Hilbert function of $I$. We also define $\mathbb{C}[z]_{I \leq s}$ to consist of all normal forms of degree $\leq s$. Since $<_{g r}$ is a graded order, the isomorphism $\mathbb{C}[z]_{I} \simeq \mathbb{C}[z] / I$ induces an isomorphism

$$
\mathbb{C}[z]_{I \leq s} \simeq(\mathbb{C}[z] / I)_{\leq s}
$$

(see [5], §9.3). This has two useful consequences:

- The Hilbert function $\operatorname{dim}(\mathbb{C}[z] / I)_{\leq s}$ is given by the number of monomials $z^{\gamma} \notin\langle\mathrm{LT}(I)\rangle$ of degree $\leq s$.

- If $r_{1} \in \mathbb{C}[z]_{I \leq s}$ and $r_{2} \in \mathbb{C}[z]_{I \leq t}$, then $r_{1} * r_{2} \in \mathbb{C}[z]_{I \leq s+t}$.

A Noether normalization $\mathbb{C}\left[z_{1}, \ldots, z_{m}\right] \subseteq \mathbb{C}[z] / I$ has the following properties. 
Proposition 2.2. Every element of $\mathbb{C}\left[z_{1}, \ldots, z_{m}\right]$ is a normal form, so that

$$
\mathbb{C}\left[z_{1}, \ldots, z_{m}\right] \subseteq \mathbb{C}[z]_{I}
$$

Furthermore, for $i=m+1, \ldots, n$, we have $z_{i}^{d_{i}} \in\langle\operatorname{LT}(I)\rangle$, where $d_{i}$ is as in (2.1).

Proof. For the second assertion of the proposition, suppose $i \in\{m+1, \ldots, n\}$ and $g_{i} \in I$ is as in (2.1). Then the definition of grevlex and the degree condition in (2.1) makes it easy to see that $\operatorname{LT}\left(g_{i}\right)=z_{i}^{d_{i}}$, which implies $z_{i}^{d_{i}} \in\langle\operatorname{LT}(I)\rangle$.

Since normal forms are known to form a subspace, it suffices to show that every monomial in $\mathbb{C}\left[z_{1}, \ldots, z_{m}\right]$ is a normal form. Let $\alpha=\left(\alpha_{1}, \alpha_{2}, \ldots, \alpha_{m}, 0, \ldots, 0\right)$, so that $z^{\alpha}=z_{1}^{\alpha_{1}} z_{2}^{\alpha_{2}} \cdots z_{m}^{\alpha_{m}}$. We want to show that $z^{\alpha} \notin\langle\operatorname{LT}(I)\rangle$.

Suppose not, i.e., $z^{\alpha} \in\langle\operatorname{LT}(I)\rangle$. We will obtain a contradiction by studying the Hilbert function. Take $z^{\gamma} \notin\langle\operatorname{LT}(I)\rangle$, where $\gamma=\left(\gamma_{1}, \ldots, \gamma_{n}\right)$. If $i \geq m+1$, then $z_{i}^{d_{i}} \in\langle\mathrm{LT}(I)\rangle$, so $z_{i}^{d_{i}}$ cannot divide $z^{\gamma}$. Hence

$$
\gamma_{i}<d_{i}, \quad \text { for all } i=m+1, \ldots, n \text {. }
$$

Furthermore, $z^{\alpha} \in\langle\operatorname{LT}(I)\rangle$, so $z^{\alpha}$ cannot divide $z^{\gamma}$. Then

$$
\gamma_{i}<\alpha_{i}, \quad \text { for some } i=1, \ldots, m \text {. }
$$

Now let

$$
L(s):=\left\{\gamma: z^{\gamma} \notin\langle\mathrm{LT}(I)\rangle,|\gamma| \leq s\right\},
$$

so that $|L(s)|=\operatorname{dim}(\mathbb{C}[z] / I)_{\leq s}$ is the Hilbert function. Also, for $i=1, \ldots, m$, let

$$
L_{i}(s)=\left\{\gamma \in L(s): \gamma_{i}<\alpha_{i} \text { and } \gamma_{m+1}<d_{m+1}, \ldots, \gamma_{n}<d_{n}\right\} .
$$

Then (2.3) and (2.4) imply that

$$
L(s) \subseteq L_{1}(s) \cup \cdots \cup L_{m}(s)
$$

Observe that

$$
\left|L_{i}(s)\right| \leq \alpha_{i} \cdot d_{m+1} \cdots d_{n} \cdot \operatorname{dim} \mathbb{C}\left[z_{1}, \ldots, \widehat{z}_{i}, \ldots, z_{m}\right]_{\leq s} .
$$

Combining this with (2.2) and (2.5), we obtain $|L(s)|=O\left(s^{m-1}\right)$. It follows that

$$
\operatorname{dim}(\mathbb{C}[z] / I)_{\leq s}=O\left(s^{m-1}\right)
$$

On the other hand, the inclusion $\mathbb{C}\left[z_{1}, \ldots, z_{m}\right] \subseteq \mathbb{C}[z] / I$ gives an inclusion

$$
\mathbb{C}\left[z_{1}, \ldots, z_{m}\right]_{\leq s} \subseteq(\mathbb{C}[z] / I)_{\leq s},
$$

and then (2.2) implies $\operatorname{dim}(\mathbb{C}[z] / I)_{\leq s} \geq \frac{1}{m !} s^{m}+O\left(s^{m-1}\right)$. This contradicts (2.6) and completes the proof.

\section{Constructing an ordered basis}

In what follows we will use the following standard notation.

Notation 3.1. Given a set of polynomials $I \subseteq \mathbb{C}\left[z_{1}, \ldots, z_{n}\right]=\mathbb{C}[z]$, write

$$
\mathbf{V}(I):=\left\{\left(a_{1}, \ldots, a_{n}\right) \in \mathbb{C}^{n}: p\left(a_{1}, \ldots, a_{n}\right)=0 \text { for all } p \in S\right\}
$$

and given a set $V \subseteq \mathbb{C}^{n}$, write

$$
\mathbf{I}(V):=\left\{p \in \mathbb{C}[z]: p\left(a_{1}, \ldots, a_{n}\right)=0 \text { for all }\left(a_{1}, \ldots, a_{n}\right) \in V\right\} .
$$


Let $V \subseteq \mathbb{C}^{n}$ be an affine algebraic variety of pure dimension $m(m \leq n)$. Here, "pure" means that all irreducible components of $V$ have dimension $m$. If we set $I:=\mathbf{I}(V) \subseteq \mathbb{C}\left[z_{1}, \ldots, z_{n}\right]$, then the coordinate ring $\mathbb{C}[V]$ of polynomial functions on $V$ satisfies

$$
\mathbb{C}[V] \simeq \mathbb{C}[z] / I \simeq \mathbb{C}[z]_{I}
$$

In what follows, we will use these isomorphisms to identify $\mathbb{C}[V]$ with $\mathbb{C}[z]_{I}$ and write $\mathbb{C}[V]=\mathbb{C}[z]_{I}$.

We will construct a special basis of $\mathbb{C}[V]$ by doing interpolation at infinity. Identify $\left(a_{1}, \ldots, a_{n}\right) \in \mathbb{C}^{n}$ with $\left[1: a_{1}: \cdots: a_{n}\right] \in \mathbb{P}^{n}$; the hyperplane at infinity is then

$$
H_{\infty}:=\left\{\left[a_{0}: a_{1}: \cdots: a_{n}\right] \in \mathbb{P}^{n}: a_{0}=0\right\}
$$

and we write $\mathbb{C}^{n} \cup H_{\infty}=\mathbb{P}^{n}$. Denote by $\bar{V} \subseteq \mathbb{P}^{n}$ the projective closure of $V$, which may be computed as follows. If $I=\mathbf{I}(V) \subseteq \mathbb{C}[z]=\mathbb{C}\left[z_{1}, \ldots, z_{n}\right]$, let

$$
I^{h}:=\left\{p^{h} \in \mathbb{C}\left[z_{0}, \ldots, z_{n}\right]: p \in I\right\},
$$

where $p(z)=\sum_{|\alpha| \leq d} c_{\alpha} z^{\alpha} \in \mathbb{C}[z]$ of degree $d$ homogenizes to

$$
p^{h}\left(z_{0}, z\right):=\sum_{|\alpha| \leq d} c_{\alpha} z_{0}^{d-|\alpha|} z^{\alpha} \in \mathbb{C}\left[z_{0}, z\right]=\mathbb{C}\left[z_{0}, z_{1}, \ldots, z_{n}\right] .
$$

Then the projective closure $\bar{V} \subseteq \mathbb{P}^{n}$ is given by

$$
\bar{V}=\mathbf{V}\left(I^{h}\right)=\left\{\left[a_{0}: \cdots: a_{n}\right] \in \mathbb{P}^{n}: p\left(a_{0}, \ldots, a_{n}\right)=0 \text { for all } p \in I^{h}\right\} .
$$

Note that $I^{h}$ is a homogeneous ideal (i.e., it is generated by homogeneous polynomials). For a homogeneous ideal $J \subseteq \mathbb{C}\left[z_{0}, \ldots, z_{n}\right]$ we will write

$$
\begin{aligned}
J_{t} & =\{p \in J: p \text { is homogeneous, } \operatorname{deg} p=t\}, \quad \text { and } \\
\left(\mathbb{C}\left[z_{0}, \ldots, z_{n}\right] / J\right)_{t} & =\mathbb{C}\left[z_{0}, \ldots, z_{n}\right]_{t} / J_{t} .
\end{aligned}
$$

We will assume that $V$ has the following properties:

(0) $V$ is pure of dimension $m$ and has degree $d$.

(1) $R:=\mathbb{C}\left[z_{1}, \ldots, z_{m}\right] \subseteq \mathbb{C}[V]$ is a Noether normalization as above.

(2) $\bar{V} \cap P$ consists of $d$ distinct points, where $\bar{V}$ is the projective closure of $V$ in $\mathbb{P}^{n}$ and $P=\mathbf{V}\left(z_{0}, \ldots, z_{m-1}\right) \subseteq \mathbb{P}^{n}$.

(3) If $\bar{V} \cap P=\left\{p_{1}, \ldots, p_{d}\right\}$, with $p_{i}=\left[0: \cdots: 0: p_{i m}: \cdots: p_{\text {in }}\right]$, then for each $i, p_{i m} \neq 0$.

Note that $\bar{V} \subseteq \mathbb{P}^{n}$ is pure of dimension $m$ and has degree $d$, while $P \subseteq \mathbb{P}^{n}$ is a linear space of dimension $n-m$ and has degree 1 . Since $\bar{V} \cap P$ is finite by property (3), Bezout's theorem implies that $\bar{V} \cap P$ consists of $d \cdot 1=d$ points counted with multiplicity. Property (3) then implies that the multiplicities of the $p_{i}$ are all one, so that

as subschemes of $\mathbb{P}^{n}$.

$$
\mathbf{V}\left(I^{h}+\left\langle z_{0}, \ldots, z_{m-1}\right\rangle\right)=\left\{p_{1}, \ldots, p_{d}\right\}
$$

It follows that the homogeneous ideals $I^{h}+\left\langle z_{0}, \ldots, z_{m-1}\right\rangle$ and $\mathbf{I}\left(\left\{p_{1}, \ldots, p_{d}\right\}\right)$ define the same subscheme of $\mathbb{P}^{n}$. Hence there is an integer $t_{0} \geq 0$ such that

$$
\begin{aligned}
\left(I^{h}+\left\langle z_{0}, \ldots, z_{m-1}\right\rangle\right)_{t} & =\left(\mathbf{I}\left(\left\{p_{1}, \ldots, p_{d}\right\}\right)\right)_{t} \\
& =\left\{f \in \mathbb{C}\left[z_{0}, \ldots, z_{n}\right]_{t}: f\left(p_{i}\right)=0, \forall i=1, \ldots, d\right\}
\end{aligned}
$$


when $t \geq t_{0}$ (see [6], II.5).

A polynomial $f \in \mathbb{C}\left[z_{0}, \ldots, z_{n}\right]_{t}$ gives a function on

$$
U_{m}=\left\{z=\left[z_{0}: z_{1}: \cdots: z_{n}\right] \in \mathbb{P}^{n}: z_{m} \neq 0\right\},
$$

via $\left[a_{0}: \cdots: a_{n}\right] \mapsto a_{m}^{-t} f\left(a_{0}, \ldots, a_{n}\right)$. It is easy to see that the computation is independent of homogeneous coordinates. For convenience this local evaluation will be denoted by $f(a)$.

Lemma 3.2. The map $\mathbb{C}\left[z_{0}, \ldots, z_{n}\right]_{t} \rightarrow \mathbb{C}^{d}$ given by $f \mapsto\left(f\left(p_{1}\right), \ldots, f\left(p_{d}\right)\right)$ is onto for $t \gg 0$.

Proof. By property (3) of (3.1), the points $p_{1}, \ldots, p_{d}$ are in the affine chart $U_{m}$ given by (3.2). For each $i=1, \ldots, d$ and $p_{i}=\left[0: \cdots: 0: 1: u_{i(m+1)}: \cdots: u_{i n}\right]$, put $q_{i}:=\left(0, \ldots, 0, u_{i(m+1)}, \ldots, u_{i n}\right) \in \mathbb{C}_{m}^{n}$, where $\mathbb{C}_{m}^{n}$ denotes affine space with coordinates $\left(z_{0}, \ldots, z_{m-1}, z_{m+1}, \ldots, z_{n}\right)$. It is standard that one can find interpolating polynomials $w_{1}, \ldots, w_{d}$ in $\mathbb{C}\left[z_{0}, \ldots, z_{m-1}, z_{m+1}, \ldots, z_{n}\right]$ such that $w_{i}\left(q_{j}\right)=\delta_{i j}$.

Pick any $t \geq \max \left(\operatorname{deg} w_{1}, \ldots, \operatorname{deg} w_{d}\right)$ and set

$$
v_{i}:=z_{m}^{t} w_{i}\left(z_{0} / z_{m}, \ldots, z_{m-1} / z_{m}, z_{m+1} / z_{m}, \ldots, z_{n} / z_{m}\right) .
$$

This is a homogeneous polynomial of degree $t$ in $z_{0}, \ldots, z_{n}$ and its evaluation on $U_{m}$ satisfies $v_{i}\left(p_{j}\right)=\delta_{i j}$. For each $i$, the polynomial $v_{i} \in \mathbb{C}\left[z_{0}, \ldots, z_{n}\right]$ evaluates to the standard basis vector $(0, \ldots, 0,1,0, \ldots, 0)=e_{i} \in \mathbb{C}^{d}$ (the 1 is in the $i$-th slot), so the map is onto.

Corollary 3.3. For $t \gg 0$, we have an exact sequence

$$
0 \longrightarrow\left(I^{h}+\left\langle z_{0}, \ldots, z_{m-1}\right\rangle\right)_{t} \longrightarrow \mathbb{C}\left[z_{0}, \ldots, z_{n}\right]_{t} \longrightarrow \mathbb{C}^{d} \longrightarrow 0
$$

Thus there are polynomials $v_{1}, \ldots, v_{d} \in \mathbb{C}\left[z_{0}, \ldots, z_{n}\right]_{t}$, unique up to elements of $\left(I^{h}+\left\langle z_{0}, \ldots, z_{m-1}\right\rangle\right)_{t}$, such that $v_{i}\left(p_{j}\right)=\delta_{i j}$.

Now fix such a $t$ and let $\mathcal{S}:=\mathbb{C}\left[z_{0}, \ldots, z_{n}\right] /\left(I^{h}+\left\langle z_{0}, \ldots, z_{m-1}\right\rangle\right)$. If we regard the polynomials $v_{1}, \ldots, v_{d}$ in the above corollary as elements of $\mathcal{S}_{t}$, then they have the following properties:

$$
v_{i}^{2}=z_{m}^{t} v_{i} \text { for all } i=1, \ldots, d ; \quad \text { and } v_{i} v_{j}=0 \text { whenever } i \neq j .
$$

Lemma 3.4. For any $\tau \geq t$, the polynomials $\left\{z_{m}^{\tau-t} v_{i}\right\}_{i=1}^{d}$ form a basis of $\mathcal{S}_{\tau}$.

Proof. The construction (3.3) applied to $\tau$ (in place of $t$ ) gives the additional powers of $z_{m}$.

When we consider the $v_{i}$ 's as polynomials in $\mathbb{C}\left[z_{0}, \ldots, z_{n}\right] /\left(I^{h}+\left\langle z_{0}\right\rangle\right)$, we have

$$
\begin{aligned}
v_{i}^{2} & =z_{m}^{t} v_{i}+\sum_{k=1}^{m-1} z_{k} H_{k}\left(z_{1}, \ldots, z_{n}\right), \\
v_{i} v_{j} & =\sum_{k=1}^{m-1} z_{k} Q_{k}\left(z_{1}, \ldots, z_{n}\right),
\end{aligned}
$$

where for each $k, H_{k}\left(z_{1}, \ldots, z_{n}\right)$ and $Q_{k}\left(z_{1}, \ldots, z_{n}\right)$ are homogeneous polynomials of degree $2 t-1$.

The next step is to translate the $v_{i}$ into polynomials $\mathbf{v}_{i}$ in $\mathbb{C}[V]$, paying careful attention to their degrees and the analogs of (3.5) and (3.6). Let $\mathbb{C}[V]_{\leq t}=\mathbb{C}[z]_{I \leq t}$ 
be the collection of normal forms of degree $\leq t$, and let $\mathbb{C}[V]_{=t}$ be those that are homogeneous of degree $t$.

Lemma 3.5. We have $\mathbb{C}[V]_{=t} \simeq \mathbb{C}[V]_{\leq t} / \mathbb{C}[V]_{\leq t-1} \simeq\left(\mathbb{C}\left[z_{0}, \ldots, z_{n}\right] /\left(I^{h}+\left\langle z_{0}\right\rangle\right)\right)_{t}$.

Proof. Writing a normal form as a sum of homogeneous components gives the direct sum decomposition $\mathbb{C}[V]_{\leq t}=\mathbb{C}[V]_{=t} \oplus \mathbb{C}[V]_{\leq t-1}$, and the first isomorphism follows immediately.

For the second, the map $p \mapsto z_{0}^{t} p\left(z_{1} / z_{0}, \ldots, z_{n} / z_{0}\right)$ induces an isomorphism

$$
\mathbb{C}[V]_{\leq t} \simeq(\mathbb{C}[z] / I)_{\leq t} \simeq\left(\mathbb{C}\left[z_{0}, z\right] / I^{h}\right)_{t}
$$

(see [5], §9.3). This isomorphism sends $\mathbb{C}[V]_{\leq t-1} \subseteq \mathbb{C}[V]_{\leq t}$ to $z_{0}\left(\mathbb{C}\left[z_{0}, z\right] / I^{h}\right)_{t-1}$, so that we get an isomorphism

$$
\mathbb{C}[V]_{\leq t} / \mathbb{C}[V]_{\leq t-1} \simeq\left(\mathbb{C}\left[z_{0}, z\right] / I^{h}\right)_{t} / z_{0}\left(\mathbb{C}\left[z_{0}, z\right] / I^{h}\right)_{t-1} \simeq\left(\mathbb{C}\left[z_{0}, z\right] /\left(I^{h}+\left\langle z_{0}\right\rangle\right)\right)_{t}
$$

Remark 3.6. Note that multiplication in $\mathbb{C}\left[z_{0}, \ldots, z_{n}\right] /\left(I^{h}+\left\langle z_{0}\right\rangle\right)$ corresponds to linear maps $\widehat{*}: \mathbb{C}[V]_{=t} \times \mathbb{C}[V]_{=s} \rightarrow \mathbb{C}[V]_{=s+t}$, where to get $p \widehat{*} q$, we compute $p * q$ (the normal form of $p q$ ) and then take the homogeneous part of degree $s+t$.

Lemma 3.7. For each $i=1, \ldots, d$, there is a polynomial $\mathbf{v}_{i} \in \mathbb{C}[V]_{=t}$ that satisfies the following equations in $\mathbb{C}[V]$ :

(1) $\mathbf{v}_{i} * \mathbf{v}_{i}=z_{m}^{t} * \mathbf{v}_{i}+\sum_{k=1}^{m-1} z_{k} * h_{k}+h_{0}$ with $\operatorname{deg}\left(h_{k}\right) \leq 2 t-1$ for each $k=$ $0, \ldots, m-1$.

(2) $\mathbf{v}_{i} * \mathbf{v}_{j}=\sum_{k=1}^{m-1} z_{k} * q_{k}+q_{0}$ if $i \neq j$ with $\operatorname{deg}\left(q_{k}\right)<2 t-1$ for each $k$.

Remark 3.8. Since $\mathbb{C}[V]$ is identified with the space $\mathbb{C}[z]_{I}$ of normal forms, the products involving $*$ in Lemma 3.7 represent multiplication of polynomials followed by reduction to normal form.

Proof. Given $v_{i} \in\left(\mathbb{C}\left[z_{0}, \ldots, z_{n}\right] /\left(I^{h}+\left\langle z_{0}\right\rangle\right)\right)_{t}$, let $\mathbf{v}_{i}$ be the element of $\mathbb{C}[V]_{=t}$ given by the isomorphism in Lemma [3.5. For each $k=1, \ldots, m-1$, let $h_{k} \in \mathbb{C}[V]_{=2 t-1}$ be the element corresponding to $H_{k} \in\left(\mathbb{C}\left[z_{0}, \ldots, z_{n}\right] /\left(I^{h}+\left\langle z_{0}\right\rangle\right)\right)_{2 t-1}$ in (3.5). Then by (3.5), the polynomial

$$
\mathbf{v}_{i} \widehat{*} \mathbf{v}_{i}-z_{m}^{t} \widehat{*} \mathbf{v}_{i}-\sum_{k=1}^{m-1} z_{k} \widehat{*} h_{k} \in \mathbb{C}[V]_{=2 t}
$$

corresponds to the zero polynomial in $\left(\mathbb{C}\left[z_{0}, \ldots, z_{n}\right] /\left(I^{h}+\left\langle z_{0}\right\rangle\right)\right)_{2 t}$, so it must be zero in $\mathbb{C}[V]_{=2 t}$. (Here, $\widehat{*}$ is as in Remark [3.6.) Thus the polynomial $h_{0}:=$ $\mathbf{v}_{i} * \mathbf{v}_{i}-z_{m}^{t} * \mathbf{v}_{i}-\sum_{k=1}^{m-1} z_{k} * h_{k}$ is in $\mathbb{C}[V]_{\leq 2 t-1}$. This proves (1).

A similar argument applied to (3.6) proves (2).

In what follows, we use the notation

$$
z^{\alpha}=z_{1}^{a_{1}} \cdots z_{m-1}^{a_{m-1}}, \quad z^{\beta}=z_{m+1}^{b_{1}} \cdots z_{n}^{b_{n}} .
$$

Define the finite set of monomials

$$
\mathcal{B}:=\left\{z_{m}^{l} z^{\beta} \notin\langle\mathrm{LT}(I)\rangle, l+|\beta| \leq t-1\right\} \subseteq \mathbb{C}[V] .
$$

Proposition 3.9. $\mathbb{C}[V]$ is spanned over $\mathbb{C}$ by the homogeneous polynomials

$$
\begin{array}{lll}
\text { (*) } & z^{\alpha} z_{m}^{l} * z^{\beta}: & \alpha \in \mathbb{Z}_{\geq 0}^{m-1}, z_{m}^{l} z^{\beta} \in \mathcal{B}, \text { and } \\
(* *) & z^{\alpha} z_{m}^{l} * \mathbf{v}_{i}: & \alpha \in \mathbb{Z}_{\geq 0}^{m-1}, l \geq 0, i=1, \ldots, d .
\end{array}
$$


Remark 3.10. Note that $z^{\alpha} z_{m}^{l}$ is a normal form by Proposition 2.2, while the products $z^{\alpha} z_{m}^{l} z^{\beta}$ and $z^{\alpha} z_{m}^{l} \mathbf{v}_{i}$ may fail to be normal forms. This explains why the proposition uses $z^{\alpha} z_{m}^{l} * z^{\beta}$ and $z^{\alpha} z_{m}^{l} * \mathbf{v}_{i}$.

Proof. To simplify the proof, we will omit the $*$ when multiplying normal forms. It suffices to show that any monomial $z^{\alpha} z_{m}^{l} z^{\beta} \notin\langle\mathrm{LT}(I)\rangle$ can be expressed as a linear combination of elements of $(*)$ and $(* *)$.

We will prove this by induction on $s=|\alpha|+l+|\beta|$. Suppose $z^{\alpha} z_{m}^{l} z^{\beta} \notin\langle\operatorname{LT}(I)\rangle$ with $s \leq t-1$. Then $|\alpha|+l+|\beta| \leq t-1$, so that $z_{m}^{l} z^{\beta} \in \mathcal{B}$. Hence the monomial is in $(*)$, which proves the base case.

Next, assume $s \geq t$ and that $\mathbb{C}[V]_{\leq s-1}$ is spanned by the polynomials $(*)$ and $(* *)$ of degree $\leq s-1$. Take $z^{\alpha} z_{m}^{l} z^{\beta} \notin\langle\operatorname{LT}(I)\rangle$ of degree $s$. No factor of this monomial is in the ideal either; in particular, $z_{m}^{l} z^{\beta} \notin\langle\operatorname{LT}(I)\rangle$. If $l+|\beta| \leq t-1$, then $z_{m}^{l} z^{\beta} \in \mathcal{B}$ and therefore $z^{\alpha} z_{m}^{l} z^{\beta}$ is an element of the form $(*)$.

Otherwise, $\tau:=l+|\beta| \geq t$. By Lemma 3.4, we have an equation

$$
z_{m}^{l} z^{\beta}=\sum_{i=1}^{d} a_{i} z_{m}^{\tau-t} v_{i}+\sum_{j=0}^{m-1} z_{j} H_{j}\left(z_{0}, z\right)+H\left(z_{0}, z\right),
$$

in $\mathbb{C}\left[z_{0}, z\right]$, where $a_{i} \in \mathbb{C}$, $\operatorname{deg} H_{j}=\tau-1$ and $H \in I^{h}$. If we dehomogenize by setting $z_{0}=1$, we obtain

$$
z_{m}^{l} z^{\beta}=\sum_{i=1}^{d} a_{i} z_{m}^{\tau-t} v_{i}+\sum_{j=1}^{m-1} z_{j} h_{j}(z)+h_{0}(z)
$$

in $\mathbb{C}[z] / I$, where $a_{i} \in \mathbb{C}$ and $\operatorname{deg} h_{j} \leq \tau-1$. We can multiply by $z^{\alpha}$ to obtain

$$
z^{\alpha} z_{m}^{l} z^{\beta}=\sum_{i=1}^{d} a_{i} z^{\alpha} z_{m}^{\tau-t} v_{i}+\sum_{j=1}^{m-1} z_{j}\left(z^{\alpha} h_{j}(z)\right)+z^{\alpha} h_{0}(z)
$$

in $\mathbb{C}[z] / I$. Using the isomorphism $\mathbb{C}[V]=\mathbb{C}[z]_{I} \simeq \mathbb{C}[z] / I$, this becomes

$$
z^{\alpha} z_{m}^{l} z^{\beta}=\sum_{i=1}^{d} a_{i} z^{\alpha} z_{m}^{\tau-t} \mathbf{v}_{i}+\sum_{j=1}^{m-1} z_{j}\left(z^{\alpha} h_{j}(z)\right)+z^{\alpha} h_{0}(z) .
$$

in $\mathbb{C}[V]$. The first sum is a linear combination of elements of the form $(* *)$. For the second sum, note that $\operatorname{deg}\left(z^{\alpha} h_{j}\right) \leq s-1$ for each $j=1, \ldots, m-1$. By the inductive hypothesis, this means that $z^{\alpha} h_{j}$ is a linear combination of terms in $(*)$ and $(* *)$, and therefore $z_{j} z^{\alpha} h_{j}$ is too, by definition. Finally, $\operatorname{deg}\left(z^{\alpha} h_{0}\right) \leq s-1$, and again by induction, $z^{\alpha} h_{0}$ is a linear combination of terms in $(*)$ and $(* *)$.

The following is an immediate corollary of the above proof.

Corollary 3.11. $\mathbb{C}[V]_{\leq s}$ is spanned over $\mathbb{C}$ by the polynomials in $(*)$ and $(* *)$ of degree $\leq s$.

Now that we have a spanning set, the next step in constructing the desired basis for $\mathbb{C}[V]$ is to show that the elements of the form $(* *)$ are linearly independent over $\mathbb{C}$. These elements are monomials in $z_{1}, \ldots, z_{m}$ multiplied by one of $\mathbf{v}_{1}, \ldots, \mathbf{v}_{d}$. Since the inclusion $\mathbb{C}\left[z_{1}, \ldots, z_{m}\right] \subseteq \mathbb{C}[V]$ makes $\mathbb{C}[V]$ into a module over $R=$ $\mathbb{C}\left[z_{1}, \ldots, z_{m}\right]$, we can verify linear independence by showing the following.

Theorem 3.12. The polynomials $\mathbf{v}_{1}, \ldots, \mathbf{v}_{d}$ generate a free $R$-submodule of $\mathbb{C}[V]$. 
Proof. We first observe that since $V$ has dimension $m$ and degree $d$, we have

$$
\operatorname{dim} \mathbb{C}[V]_{\leq s}=\frac{d}{m !} s^{m}+O\left(s^{m-1}\right)
$$

(see e.g. [5], §9.3). Now let $M:=\sum_{i=1}^{d} R \mathbf{v}_{i}$ and $N:=\sum_{\mathcal{B}} \mathbb{C}\left[z_{1}, \ldots, z_{m-1}\right] z_{m}^{l} z^{\beta}$, and for $s \geq t$ define

$$
M_{\leq s}:=\sum_{i=1}^{d} R_{\leq s-t} \mathbf{v}_{i}, \quad N_{\leq s}:=\sum_{\mathcal{B}} \mathbb{C}\left[z_{1}, \ldots, z_{m-1}\right]_{\leq s-l-|\beta|} z_{m}^{l} z^{\beta} .
$$

The corollary implies $\mathbb{C}[V]_{\leq s}=M_{\leq s}+N_{\leq s}$. Using (2.2), one easily obtains

$$
\operatorname{dim} N_{\leq s} \leq \frac{|\mathcal{B}|}{(m-1) !} s^{m-1}+O\left(s^{m-2}\right) .
$$

Combining this with (3.8) and $\mathbb{C}[V]_{\leq s}=M_{\leq s}+N_{\leq s}$ yields

$$
\operatorname{dim} M_{\leq s}=\frac{d}{m !} s^{m}+O\left(s^{m-1}\right) .
$$

Suppose there is a nontrivial relation

$$
f_{1} \mathbf{v}_{1}+\cdots+f_{d} \mathbf{v}_{d}=0, \quad f_{i} \in R, \text { not all } f_{i}=0 .
$$

Let $D=\max \left\{\operatorname{deg} f_{1}, \ldots, \operatorname{deg} f_{d}\right\}$ and take a large integer $s \geq t+D$. There is an exact sequence

$$
0 \longrightarrow K_{s} \longrightarrow R_{\leq s-t}^{d} \stackrel{\varphi_{s}}{\longrightarrow} M_{\leq s} \longrightarrow 0
$$

where $\varphi_{s}: R_{\leq s-t}^{d} \rightarrow M_{\leq s-t}$ is given by $\varphi\left(g_{1}, \ldots, g_{d}\right)=\sum_{i} g_{i} \mathbf{v}_{i}$ and $K_{s}:=\operatorname{ker} \varphi_{s}$. We have $R_{s-t-D} \cdot\left(f_{1}, \ldots, f_{d}\right) \subseteq R_{\leq s-t}^{d}$, so by (3.9),

$$
R_{s-t-D}\left(f_{1}, \ldots, f_{d}\right) \subseteq K_{s} .
$$

Since $\left(f_{1}, \ldots, f_{d}\right) \neq(0, \ldots, 0)$ we have $K_{s} \neq 0$ and so $\operatorname{dim} K_{s} \geq \operatorname{dim} R_{s-t-D}$. Thus

$$
\operatorname{dim} R_{\leq s-t}^{d}=\operatorname{dim} M_{\leq s}+\operatorname{dim} K_{s} \geq \operatorname{dim} M_{\leq s}+\operatorname{dim} R_{s-t-D} .
$$

A Hilbert function calculation then gives the inequality

$$
\frac{d}{m !} s^{m}+O\left(s^{m-1}\right) \geq\left(\frac{d}{m !} s^{m}+O\left(s^{m-1}\right)\right)+\left(\frac{1}{m !}(s-t-D)^{m}+O\left(s^{m-1}\right)\right),
$$

so that $\frac{1}{m !} s^{m} \leq O\left(s^{m-1}\right)$, a contradiction. This says that no equation of the form (3.9) can hold, and so $\mathbf{v}_{1}, \ldots, \mathbf{v}_{d}$ are free over $R$.

We now construct the sought-after ordered basis for $\mathbb{C}[V]$.

Definition 3.13. The polynomials given by $(*)$ and $(* *)$ span $\mathbb{C}[V]$ by Proposition [3.9, and those from $(* *)$ are linearly independent by Theorem 3.12 . We first create a basis of $\mathbb{C}[V]$ by adjoining a sufficient number of elements of the form $(*)$ to those of the form $(* *)$. List those of the form $(*)$ in grevlex order and discard any monomial that is linearly dependent with respect to elements of the form $(* *)$ together with previous elements of $(*)$; otherwise keep it. This yields the basis $\mathcal{C}$ of $\mathbb{C}[V]$. We define an ordering $\prec$ on $\mathcal{C}$ as follows. First, order the elements by total degree; then for a fixed degree $s$,

- let elements of $(*)$ precede elements of $(* *)$;

- let $z^{\alpha} z_{m}^{l} * \mathbf{v}_{i} \prec z^{\widehat{\alpha}} z_{m}^{\widehat{l}} * \mathbf{v}_{j}$ if $z^{\alpha} z_{m}^{l}$ precedes $z^{\widehat{\alpha}} z_{m}^{\widehat{l}}$ according to grevlex;

- let $z^{\alpha} z_{m}^{l} * \mathbf{v}_{i} \prec z^{\alpha} z_{m}^{l} * \mathbf{v}_{j}$ if $i<j$; and

- let elements of the form $(*)$ be ordered according to grevlex. 
It is easy to see that the elements of $\mathcal{C}$ of degree $\leq s$ form a basis of $\mathbb{C}[V]_{<s}$. The Chebyshev constants defined in Section 5 will use the ordered basis of $\mathbb{C}[\bar{V}]$ given in Definition 3.13 .

We conclude this section by computing some examples of $\mathcal{C}$ and $\prec$.

Example 3.14. Let $V=\left\{z=\left(z_{1}, \ldots, z_{n}\right) \in \mathbb{C}^{n}: z_{m+1}=z_{m+2}=\cdots=z_{n}=0\right\}$. The Noether normalization is the identity, $\mathbb{C}\left[z_{1}, \ldots, z_{m}\right]=\mathbb{C}[V]$, and in the notation of (3.1), $\bar{V} \cap P=\{[0: \cdots: 0: 1: 0: \cdots: 0]\}$, where the 1 is in the $m$-th slot. We take $v_{1}=\mathbf{v}_{1}=1$ (so $\left.t=0\right)$. The basis $\mathcal{C}$ consists of the monomials in $\mathbb{C}\left[z_{1}, \ldots, z_{m}\right]$, which are elements of the form $(* *)$, ordered by grevlex. There are no elements of the form $(*)$ in this case.

Example 3.15. Let $V$ be the complexified sphere in $\mathbb{C}^{3}$, i.e., the algebraic surface given by the equation $z_{1}^{2}+z_{2}^{2}+z_{3}^{2}=1$. A basis of $\mathbb{C}[V]$ is given by all monomials not in $\left\langle z_{3}^{2}\right\rangle$, i.e.

$$
1, z_{1}, z_{2}, z_{3}, z_{1}^{2}, z_{1} z_{2}, z_{1} z_{3}, z_{2}^{2}, z_{2} z_{3}, z_{1}^{3}, \ldots
$$

The Noether normalization is $\mathbb{C}\left[z_{1}, z_{2}\right] \subseteq \mathbb{C}[V]$.

In $\mathbb{P}^{3}, \bar{V}$ is given by all points $\left[z_{0}: z_{1}: z_{2}: z_{3}\right]$ satisfying $z_{1}^{2}+z_{2}^{2}+z_{3}^{2}=z_{0}^{2}$, and $P=\left\{z_{0}=z_{1}=0\right\}$. The points of $\bar{V} \cap P$ are then $p_{1}=[0: 0: 1:-i]$ and $p_{2}=[0: 0: 1: i]$. Thus (3.1) is satisfied.

Interpolating polynomials are $v_{1}=\frac{1}{2}\left(z_{2}+i z_{3}\right)$ and $v_{2}=\frac{1}{2}\left(z_{2}-i z_{3}\right)$. In this case $t=1$ so that $\mathbf{v}_{1}=v_{1}$ and $\mathbf{v}_{2}=v_{2}$. The first few elements of the basis $\mathcal{C}$, ordered by $\prec$, are

$$
1, z_{1}, \mathbf{v}_{1}, \mathbf{v}_{2}, z_{1}^{2}, z_{1} \mathbf{v}_{1}, z_{1} \mathbf{v}_{2}, z_{2} \mathbf{v}_{1}, z_{2} \mathbf{v}_{2}, z_{1}^{3}, \ldots
$$

Basis elements of the form $(*)$ are $z_{1}^{k}$ while those of the form $(* *)$ are $z_{1}^{\alpha_{1}} z_{2}^{\alpha_{2}} \mathbf{v}_{i}$. (Note that since $l<t=1$, no factors of the form $z_{2}^{l}$ appear in $(*)$ ).

Example 3.16. When $V=\mathbf{V}(f) \subseteq \mathbb{C}^{n}$ is a hypersurface given by $f \in \mathbb{C}\left[z_{1}, \ldots, z_{n}\right]$, we can generalize Example 3.15 by computing the basis $\mathcal{C}$ rather explicitly. We assume that $f$ is a product of distinct irreducible polynomials, so that $I=\mathbf{I}(V)=$ $\langle f\rangle$. We also assume that $\operatorname{LT}(f)=z_{n}^{d}$ where $d=\operatorname{deg}(f)$. This ensures that $\mathbb{C}\left[z_{1}, \ldots, z_{n-1}\right] \subseteq \mathbb{C}[V]$ is a Noether normalization.

Let $F:=f^{h} \in \mathbb{C}\left[z_{0}, \ldots, z_{n}\right]$ be the homogenization of $f$; then in $\mathbb{P}^{n}, \bar{V}=\mathbf{V}(F)$ and $I^{h}=\langle F\rangle$. If the properties (3.1) hold, then $\mathbf{V}\left(F, z_{0}, \ldots, z_{n-2}\right) \subseteq \mathbb{P}^{n}$ consists of $d$ distinct points, all with $z_{n-1} \neq 0$, given by $\left[0: \cdots: 1: \beta_{i}\right]$ for $i=1, \ldots, d$.

Separating the terms of $F$ containing only the variables $z_{n-1}, z_{n}$ from the others, we have

$$
F(z)=G\left(z_{n-1}, z_{n}\right)+\sum_{l=0}^{n-2} z_{l} H_{l}\left(z_{0}, \ldots, z_{n}\right),
$$

where $\operatorname{deg}(G)=d$ and $\operatorname{deg} H_{l}=d-1$ for each $l=0, \ldots, n-2$. Thus $G\left(1, \beta_{i}\right)=0$ for $i=1, \ldots, d$.

In the notation of earlier in the section, we have

$$
\begin{aligned}
\mathcal{S} & =\mathbb{C}\left[z_{0}, \ldots, z_{n}\right] /\left(I^{h}+\left\langle z_{0}, \ldots, z_{n-2}\right\rangle\right)=\mathbb{C}\left[z_{0}, \ldots, z_{n}\right] /\left\langle F(z), z_{0}, \ldots, z_{n-2}\right\rangle \\
& =\mathbb{C}\left[z_{0}, \ldots, z_{n}\right] /\left\langle G\left(z_{n-1}, z_{n}\right), z_{0}, \ldots, z_{n-2}\right\rangle \\
& \simeq \mathbb{C}\left[z_{n-1}, z_{n}\right] /\left\langle G\left(z_{n-1}, z_{n}\right)\right\rangle,
\end{aligned}
$$


where the second line uses (3.10) and the third uses the map

$$
p\left(z_{0}, z_{1}, \ldots, z_{n}\right) \mapsto p\left(0, \ldots, 0, z_{n-1}, z_{n}\right) .
$$

We factor $G\left(z_{n-1}, z_{n}\right)=\prod_{i=1}^{d}\left(z_{n}-\beta_{i} z_{n-1}\right)=\prod_{i=1}^{d} l_{i}\left(z_{n-1}, z_{n}\right)$. Note that $\beta_{i} \neq \beta_{j}$ if $i \neq j$. For each $i=1, \ldots, d$, define

$$
v_{i}\left(z_{n-1}, z_{n}\right)=\prod_{j \neq i} \frac{l_{j}\left(z_{n-1}, z_{n}\right)}{l_{j}\left(1, \beta_{i}\right)} .
$$

Then $\operatorname{deg}\left(v_{i}\right)=d-1$ for each $i$, and clearly

$$
v_{i}\left(1, \beta_{j}\right)= \begin{cases}0 & \text { if } j \neq i \\ 1 & \text { if } j=i .\end{cases}
$$

Note that when $f=z_{1}^{2}+z_{2}^{2}+z_{3}^{2}-1$ as in Example 3.15, we have the points $[0: 0: 1:-i]$ and $[0: 0: 1: i]$. Then $G=z_{2}^{2}+z_{3}^{2}=\left(z_{3}+i z_{2}\right)\left(z_{3}-i z_{2}\right)=l_{1} l_{2}$ and the formula for $v_{1}$ reduces to

$$
v_{1}=\frac{l_{2}\left(z_{2}, z_{3}\right)}{l_{2}(1,-i)}=\frac{z_{3}-i z_{2}}{-2 i}=\frac{1}{2}\left(z_{2}+i z_{3}\right),
$$

in agreement with Example 3.15. The formula for $v_{2}$ works similarly.

By (3.12), $v_{1}, \ldots, v_{d}$ satisfy Lemma 3.4 with $t=d-1$. Since the $v_{i}$ only involve $z_{n-1}, z_{n}$ and are normal forms with respect to grevlex (having degree $\leq d-1$ in $z_{n}$ ), we can take $\mathbf{v}_{i}=v_{i}$ in Lemma 3.7. Thus $\mathbf{v}_{1}, \ldots, \mathbf{v}_{d}$ are defined by (3.11) and have degree $d-1$.

The next step is to identify the set $\mathcal{B}$ from (3.7). Since $m=n-1$, the monomials $z^{\alpha}$ and $z^{\beta}$ from Proposition 3.9 are

$$
z^{\alpha}=z_{1}^{a_{1}} \cdots z_{n-2}^{a_{n-2}}, \quad z^{\beta}=z_{n}^{b} .
$$

In this notation, a monomial in $z_{1}, \ldots, z_{n}$ is written $z^{\alpha} z_{n-1}^{l} z_{n}^{b}$. Since the $\mathbf{v}_{i}$ have degree $t=d-1$ and $\langle\operatorname{LT}(I)\rangle=\langle\operatorname{LT}(f)\rangle=\left\langle z_{n}^{d}\right\rangle$, it follows that (3.7) becomes

$$
\mathcal{B}=\left\{z_{n-1}^{l} z_{n}^{b} \notin\left\langle z_{n}^{d}\right\rangle: l+b \leq d-2\right\}=\left\{z_{n-1}^{l} z_{n}^{b}: l+b \leq d-2\right\} .
$$

Hence the collections $(*)$ and $(* *)$ from Proposition 3.9 are

$$
\begin{aligned}
(*) z^{\alpha} z_{n-1}^{l} z_{n}^{b}: & \alpha \in \mathbb{Z}_{\geq 0}^{n-2}, l+b \leq d-2, \text { and } \\
(* *) z^{\alpha} z_{n-1}^{l} \mathbf{v}_{i}: & \alpha \in \mathbb{Z}_{\geq 0}^{n-2}, l \geq 0, i=1, \ldots, d .
\end{aligned}
$$

These products are all normal forms, so no $*$ is needed in the multiplications.

The nicest feature of the hypersurface case is that the basis $\mathcal{C}$ consists precisely of the polynomials in (3.13). They span by Proposition 3.9, so we only need to prove linear independence. The polynomials in $(* *)$ are linearly independent by Theorem 3.12 , and those in $(*)$ are linearly independent since they are normal-form monomials. Hence it remains to study an equation of the form

$$
\text { linear combination of } z^{\alpha} z_{n-1}^{l} z_{n}^{b}=\text { linear combination of } z^{\alpha} z_{n-1}^{l} \mathbf{v}_{i} \text {. }
$$

The left-hand side has degree $\leq d-2$ in $z_{n-1}, z_{n}$ and the right-hand side has degree $\geq d-1$. This forces the linear combinations to be trivial, and linear independence follows.

To summarize: when $V=\mathbf{V}(f)$ is a hypersurface of degree $d$, the $\mathbf{v}_{i}$ 's are polynomials of degree $d-1$ that we can compute explicitly in terms of $f$, and the elements of $(*)$ consist of all monomials $z_{1}^{\alpha_{1}} \cdots z_{n-1}^{\alpha_{n-1}} z_{n}^{\alpha_{n}}$ with $\alpha_{n-1}+\alpha_{n} \leq d-2$. 


\section{WEAKLY SUBMULTIPLICATIVE FUNCTIONS}

In [3], Bloom and Levenberg observed that the main properties of Zaharjuta's directional Chebyshev constants followed from the submultiplicative property of sup norms of Chebyshev polynomials, and could be recast rather abstractly as properties of submultiplicative functions on integer tuples. We verify here that these properties still hold under slightly weaker conditions. The arguments are those of Zaharjuta's paper [1] with minor adjustments. We will apply these results concretely in the next section.

Definition 4.1. Let $m$ be a positive integer. A non-negative function $Y: \mathbb{Z}_{\geq 0}^{m} \rightarrow$ $\mathbb{R}_{\geq 0}$ is said to be weakly submultiplicative if there is a finite subset $\mathcal{F}$ of $\mathbb{Z}_{\geq 0}^{m}$ such that:

For all $\alpha, \beta \in \mathbb{Z}_{\geq 0}^{m}$ there exists $\gamma \in \mathcal{F}$ such that $Y(\alpha+\beta+\gamma) \leq Y(\alpha) Y(\beta)$.

$Y$ has subexponential growth if for some $C, r>0$ we have $Y(\alpha) \leq C r^{|\alpha|}$ for all $\alpha$.

Remark 4.2 (cf. 3]). When $Y(\alpha+\beta) \leq Y(\alpha) Y(\beta)$, i.e., $\mathcal{F}=\{(0, \ldots, 0)\}, Y$ is called submultiplicative. A submultiplicative function automatically has subexponential growth: if $\alpha=\left(\alpha_{1}, \ldots, \alpha_{m}\right)$ then

$$
Y(\alpha)=Y\left(\sum_{k=1}^{m} \alpha_{k} e_{k}\right) \leq \prod_{k=1}^{m} Y\left(e_{k}\right)^{\alpha_{k}} \leq r^{|\alpha|},
$$

where $e_{k}$ is the $k$-th coordinate vector and $r=\max _{k} Y\left(e_{k}\right)$. It seems that weak submultiplicativity should also imply subexponential growth, but the above argument runs into some technical difficulties.

Let

$$
\Sigma_{m}:=\left\{\theta=\left(\theta_{1}, \ldots, \theta_{m}\right) \in \mathbb{R}^{m}: \theta_{i} \geq 0 \forall i, \sum_{i} \theta_{i}=1\right\}
$$

denote the simplex in $\mathbb{R}^{m}$, and let $\Sigma_{m}^{\circ}:=\left\{\theta \in \Sigma_{m}: \theta_{i}>0 \forall i\right\}$ be its interior.

Lemma 4.3. Let $Y: \mathbb{Z}_{\geq 0}^{m} \rightarrow \mathbb{R}_{\geq 0}$ be weakly submultiplicative with subexponential growth. For all $\theta \in \Sigma_{m}^{\circ}$, the limit $T(\theta):=\lim _{\substack{|\alpha| \rightarrow \infty \\ \frac{\alpha}{|\alpha|} \rightarrow \theta}} Y(\alpha)^{\frac{1}{|\alpha|}}$ exists.

Proof. Let $\left\{\alpha_{(j)}\right\}$ and $\left\{\tilde{\alpha}_{(j)}\right\}$ be sequences in $\mathbb{Z}_{\geq 0}^{m}$ such that $\frac{\alpha_{(j)}}{\left|\alpha_{(j)}\right|}, \frac{\tilde{\alpha}_{(j)}}{\left|\tilde{\alpha}_{(j)}\right|} \rightarrow \theta$ as $j \rightarrow \infty$ and

$$
\begin{aligned}
& \lim _{j \rightarrow \infty} Y\left(\alpha_{(j)}\right)^{\frac{1}{\alpha \alpha}(j)}=\liminf _{|\alpha| \rightarrow \infty, \frac{\alpha}{|\alpha|} \rightarrow \theta} Y(\alpha)^{\frac{1}{|\alpha|}}:=L_{1}, \\
& \lim _{j \rightarrow \infty} Y\left(\tilde{\alpha}_{(j)}\right)^{\frac{1}{\mid \tilde{\alpha}_{(j)}}}=\operatorname{limiup}_{|\alpha| \rightarrow \infty, \frac{\alpha}{|\alpha|} \rightarrow \theta} Y(\alpha)^{\frac{1}{|\alpha|}}:=L_{2} .
\end{aligned}
$$

To prove the lemma it is sufficient to show that $L_{2} \leq L_{1}$. By passing to subsequences we may assume that $\frac{\left|\tilde{\alpha}_{(j)}\right|}{\left|\alpha_{(j)}\right|} \rightarrow \infty$ as $j \rightarrow \infty$.

Let $q_{j}$ denote the largest non-negative integer for which all the components of $r_{(j)}:=\tilde{\alpha}_{(j)}-q_{j} \alpha_{(j)}$ are non-negative. We claim that

$$
\frac{q_{j}\left|\alpha_{(j)}\right|}{\left|\tilde{\alpha}_{(j)}\right|} \rightarrow 1, \frac{\left|r_{(j)}\right|}{\left|\tilde{\alpha}_{(j)}\right|} \rightarrow 0 \quad \text { as } j \rightarrow \infty
$$


Write $\alpha_{(j)}=\left|\alpha_{(j)}\right|\left(\theta+\epsilon_{(j)}\right)$ and $\tilde{\alpha}_{(j)}=\left|\tilde{\alpha}_{(j)}\right|\left(\theta+\tilde{\epsilon}_{(j)}\right)$ where $\epsilon_{(j)}, \tilde{\epsilon}_{(j)} \rightarrow 0$ as $j \rightarrow \infty$. A calculation in components shows that

$$
\tilde{\alpha}_{(j) \nu}=\frac{\left|\tilde{\alpha}_{(j)}\right|}{\left|\alpha_{(j)}\right|}\left(1+\frac{\left|\alpha_{(j)}\right|}{\alpha_{(j) \nu}}\left(\tilde{\epsilon}_{(j) \nu}-\epsilon_{(j) \nu}\right)\right) \alpha_{(j) \nu} \quad \text { for each } \nu=1, \ldots, m,
$$

where we write $\alpha_{(j)}=\left(\alpha_{(j) 1}, \ldots, \alpha_{(j) m}\right)$, etc. For any $\nu$, we have

$$
\frac{\left|\alpha_{(j)}\right|}{\alpha_{(j) \nu}}\left(\tilde{\epsilon}_{(j) \nu}-\epsilon_{(j) \nu}\right) \longrightarrow \frac{1}{\theta_{\nu}}(0-0)=0 \quad \text { as } j \rightarrow \infty .
$$

(Here we use the fact that $\theta \in \Sigma_{m}^{\circ}$, so $\theta_{\nu} \neq 0$.) This says that given $\epsilon>0$, the quantity in parentheses on the right-hand side of (4.2) exceeds $1-\epsilon$ for all $\nu$ when $j$ is sufficiently large. The definition of $q_{j}$ then implies that

$$
q_{j} \geq \frac{\left|\tilde{\alpha}_{(j)}\right|}{\left|\alpha_{(j)}\right|}(1-\epsilon)-1
$$

and hence $\frac{q_{j}\left|\alpha_{(j)}\right|}{\left|\tilde{\alpha}_{(j)}\right|} \geq 1-\epsilon-\frac{\left|\alpha_{(j)}\right|}{\left|\tilde{\alpha}_{(j)}\right|} \longrightarrow 1-\epsilon$ as $j \rightarrow \infty$. On the other hand, $\frac{q_{j}\left|\alpha_{(j)}\right|}{\left|\tilde{\alpha}_{(j)}\right|} \leq 1$ for all $j$. Since $\epsilon$ is arbitrary, (4.1) follows.

Let $c:=\max \left\{\gamma_{\nu}: \nu \in\{1, \ldots, m\},\left(\gamma_{1}, \ldots, \gamma_{m}\right) \in \mathcal{F}\right\}$, and let $s_{j}$ be the largest non-negative integer such that

$$
s_{j}\left(\alpha_{(j) \nu}+c\right) \leq q_{j} \alpha_{(j) \nu} \text { for all } \nu=1, \ldots, m .
$$

Using this, there exists $\tilde{r}_{(j)} \in \mathbb{Z}_{\geq 0}^{m}$ such that

$$
Y\left(\tilde{\alpha}_{(j)}\right)=Y\left(q_{j} \alpha_{(j)}+r_{(j)}\right)=Y\left(s_{j} \alpha_{(j)}+s_{j} \gamma_{(j)}+\tilde{r}_{(j)}\right)
$$

where $\gamma_{(j)} \in \mathcal{F}$ satisfies $Y\left(2 \alpha_{(j)}+\gamma_{(j)}\right) \leq Y\left(\alpha_{(j)}\right)^{2}$. It is easy to see that $\frac{\left|q_{j}\right|}{\left|s_{j}\right|} \rightarrow 1$, and hence (4.1) holds with $q_{j}, r_{(j)}$ replaced by $s_{j}, \tilde{r}_{(j)}$. Finally,

$$
\begin{aligned}
Y\left(\tilde{\alpha}_{(j)}\right)^{\frac{1}{\tilde{\alpha}_{(j)}}} & =Y\left(s_{j} \alpha_{(j)}+s_{j} \gamma_{(j)}+\tilde{r}_{(j)}\right)^{\frac{1}{\tilde{\alpha}_{(j)} \mid}} \\
& \leq\left(Y\left(\alpha_{(j)}\right)^{s_{j}} Y\left(\tilde{r}_{(j)}\right)\right)^{\frac{1}{\left|\frac{1}{\alpha}(j)\right|}} \leq\left(Y\left(\alpha_{(j)}\right)^{\left.\frac{1}{\mid \alpha(j)}\right)^{\frac{s_{j}|\alpha(j)|}{\tilde{\alpha}_{(j)}}}} C^{\frac{1}{\tilde{\alpha}_{(j)} \mid}} r^{\frac{|\tilde{r}(j)|}{\tilde{\alpha}(j) \mid}}\right.
\end{aligned}
$$

where $C, r$ are as in Definition 4.1. Taking the limit as $j \rightarrow \infty$ of the first and last expressions yields $L_{2} \leq L_{1}$. This completes the proof.

Recall that a positive real-valued function $f$ on a convex set $C \subseteq \mathbb{R}^{n}$ is said to be logarithmically convex if $f((1-t) a+t b) \leq f(a)^{1-t} f(b)^{t}$ for all $a, b \in C$; equivalently, $\log (f)$ is convex.

Lemma 4.4. The function $\theta \mapsto T(\theta)$, defined as in the previous lemma, is uniformly bounded and logarithmically convex on $\Sigma_{m}^{\circ}$ (and hence continuous).

Proof. Boundedness follows easily from subexponential growth: if $Y(\alpha) \leq C r^{|\alpha|}$ for all $\alpha \in \mathbb{Z}_{\geq 0}^{m}$ then $T(\theta) \leq r$ for all $\theta \in \Sigma_{m}^{\circ}$.

To prove logarithmic convexity, fix $\theta, \tilde{\theta} \in \Sigma_{m}^{\circ}$ and $t \in(0,1)$. Let $\alpha_{(j)}, \alpha_{(j)}$ satisfy $\frac{\alpha_{(j)}}{\left|\alpha_{(j)}\right|} \rightarrow \theta, \frac{\tilde{\alpha}_{(j)}}{\left|\tilde{\alpha}_{(j)}\right|} \rightarrow \tilde{\theta}$ as $j \rightarrow \infty$ and $\left|\alpha_{(j)}\right|=\left|\tilde{\alpha}_{(j)}\right|=: a_{j}$ for each $j$. Let $q_{j}, \tilde{q}_{j}$ be positive integers such that $\frac{q_{j}}{q_{j}+\tilde{q}_{j}} \rightarrow t$ as $j \rightarrow \infty$. 
For each $j$ there exist $\beta_{(j)}, \gamma_{(j)}, \tilde{\gamma}_{(j)} \in \mathcal{F}$ such that

$$
\begin{aligned}
& Y\left(q_{j} \alpha_{(j)}+\tilde{q}_{j} \tilde{\alpha}_{(j)}+\beta_{(j)}+\left(q_{j}-1\right) \gamma_{(j)}+\left(\tilde{q}_{j}-1\right) \tilde{\gamma}_{(j)}\right) \\
& \quad \leq Y\left(q_{j} \alpha_{(j)}+\left(q_{j}-1\right) \gamma_{(j)}\right) Y\left(\tilde{q}_{j} \tilde{\alpha}_{(j)}+\left(\tilde{q}_{j}-1\right) \tilde{\gamma}_{(j)}\right) \leq Y\left(\alpha_{(j)}\right)^{q_{j}} Y\left(\tilde{\alpha}_{(j)}\right)^{\tilde{q}_{j}} .
\end{aligned}
$$

Let $\zeta_{(j)}:=q_{j} \alpha_{(j)}+\tilde{q}_{j} \tilde{\alpha}_{(j)}+\beta_{(j)}+\left(q_{j}-1\right) \gamma_{(j)}+\left(\tilde{q}_{j}-1\right) \tilde{\gamma}_{(j)}$. Since $\mathcal{F}$ is bounded, it is easy to see that $\frac{\left|\zeta_{(j)}\right|}{\left|q_{j} \alpha_{(j)}+\tilde{q}_{j} \tilde{\alpha}_{(j)}\right|} \rightarrow 1$ as $j \rightarrow \infty$ and

$\lim _{j \rightarrow \infty} \frac{\zeta_{(j)}}{\left|\zeta_{(j)}\right|}=\lim _{j \rightarrow \infty} \frac{q_{j} \alpha_{(j)}+\tilde{q}_{j} \tilde{\alpha}_{(j)}}{\left|q_{j} \alpha_{(j)}+\tilde{q}_{j} \tilde{\alpha}_{(j)}\right|}=\lim _{j \rightarrow \infty} \frac{q_{j} \alpha_{(j)}}{\left(q_{j}+\tilde{q}_{j}\right) a_{j}}+\frac{\tilde{q}_{j} \tilde{\alpha}_{(j)}}{\left(q_{j}+\tilde{q}_{j}\right) a_{j}}=t \theta+(1-t) \tilde{\theta}$.

Hence

$$
\begin{aligned}
T(t \theta+(1-t) \tilde{\theta}) & =\lim _{j \rightarrow \infty} Y\left(\zeta_{(j)}\right)^{\frac{1}{\mid \zeta_{(j)}}} \\
& =\lim _{j \rightarrow \infty} Y\left(\zeta_{(j)}\right)^{\frac{1}{\mid q_{j} \alpha_{(j)}+\tilde{q}_{j} \tilde{\alpha}(j)}} \\
& \leq \lim _{j \rightarrow \infty}\left(Y\left(\alpha_{(j)}\right)^{\left.\frac{1}{\mid \alpha(j)}\right)^{\frac{q_{j}}{q_{j}+\tilde{q}_{j}}}}\left(Y\left(\tilde{\alpha}_{(j)}\right)^{\frac{1}{\mid \tilde{\alpha}_{(j)}}}\right)^{\frac{\tilde{q}_{j}}{q_{j}+\tilde{q}_{j}}}=T(\theta)^{t} T(\tilde{\theta})^{1-t},\right.
\end{aligned}
$$

which concludes the proof.

Given $b \in \partial \Sigma_{m}=\Sigma_{m} \backslash \Sigma_{m}^{\circ}$, define

$$
T^{-}(b):=\liminf _{|\alpha| \rightarrow \infty, \frac{\alpha}{|\alpha|} \rightarrow b} Y(\alpha)^{\frac{1}{|\alpha|}} .
$$

Lemma 4.5. Let $b \in \partial \Sigma_{m}$. Then

$$
T^{-}(b)=\liminf _{\theta \rightarrow b, \theta \in \Sigma_{m}^{\circ}} T(\theta) .
$$

Proof. Let $\left\{\theta_{(j)}\right\}_{j \geq 1}$ be a sequence of points in $\Sigma_{m}^{0}$ with $\theta_{(j)} \rightarrow b$ as $j \rightarrow \infty$, and for each $j$ choose $\alpha_{(j)}$ such that

$$
\left|\frac{\alpha_{(j)}}{\left|\alpha_{(j)}\right|}-\theta_{(j)}\right|<1 / j, \quad\left|Y\left(\alpha_{(j)}\right)^{\frac{1}{\alpha_{(j)}}}-T\left(\theta_{(j)}\right)\right|<1 / j .
$$

Then $\frac{\alpha_{(j)}}{\left|\alpha_{(j)}\right|} \rightarrow b$ as $j \rightarrow \infty$, so

$$
T^{-}(b) \leq \liminf _{j \rightarrow \infty} Y\left(\alpha_{(j)}\right)^{\frac{1}{\alpha_{(j)}}} \leq \liminf _{j \rightarrow \infty}\left(T\left(\theta_{(j)}\right)+1 / j\right)=\liminf _{j \rightarrow \infty} T\left(\theta_{(j)}\right) .
$$

Hence $T^{-}(b) \leq \liminf _{\theta \rightarrow b, \theta \in \Sigma^{\circ}} T(\theta)$ since the sequence $\theta_{(j)}$ was arbitrary.

It remains to prove the reverse inequality. Let $\sigma=\left(\sigma_{1}, \ldots, \sigma_{m}\right)$ satisfy $\sigma_{\nu}>0$ for each $\nu$; then $\frac{b+\sigma}{1+|\sigma|} \in \Sigma_{m}^{\circ}$. We will show that

$$
T\left(\frac{b+\sigma}{1+|\sigma|}\right) \leq r^{\frac{|\sigma|}{1+|\sigma|}} T^{-}(b)^{\frac{1}{1+|\sigma|}} .
$$

(Here $r$ is as in Definition 4.1.)

Choose sequences $\alpha_{(j)}, \ell_{(j)}$ in $\mathbb{Z}_{\geq 0}^{m}$ such that $\left|\alpha_{(j)}\right| \rightarrow \infty$ and

$$
\frac{\alpha_{(j)}}{\left|\alpha_{(j)}\right|} \rightarrow b \text { with } Y\left(\alpha_{(j)}\right)^{\frac{1}{\alpha_{(j)}}} \rightarrow T^{-}(b), \quad \text { and } \frac{\ell_{(j)}}{\left|\alpha_{(j)}\right|} \rightarrow \sigma .
$$

Since $Y$ is weakly submultiplicative with subexponential growth,

$$
Y\left(\ell_{(j)}+\alpha_{(j)}+\gamma_{(j)}\right) \leq Y\left(\ell_{(j)}\right) Y\left(\alpha_{(j)}\right) \leq C r^{\mid \ell_{(j)}} Y\left(\alpha_{(j)}\right)
$$

for appropriate $\gamma_{(j)} \in \mathcal{F}$. 
We compute $\frac{\ell_{(j)}}{\left|\alpha_{(j)}+\ell_{(j)}\right|} \rightarrow \frac{\sigma}{1+|\sigma|}$ and $\frac{\alpha_{(j)}}{\left|\alpha_{(j)}+\ell_{(j)}\right|} \rightarrow \frac{b}{1+|\sigma|}$ as $j \rightarrow \infty$. Since $\mathcal{F}$ is bounded we also have $\frac{\gamma_{(j)}}{\left|\ell_{(j)}+\alpha_{(j)}+\gamma_{(j)}\right|} \rightarrow(0, \ldots, 0)$ and $\frac{\left|\ell_{(j)}+\alpha_{(j)}\right|}{\left|\ell_{(j)}+\alpha_{(j)}+\gamma_{(j)}\right|} \rightarrow 1$. The inequality (4.5) then yields (4.4) by a similar limiting process as detailed in the previous lemmas. Finally, using (4.4), we have

$$
\liminf _{\theta \rightarrow b, \theta \in \Sigma_{m}^{\circ}} T(\theta) \leq \liminf _{\substack{|\sigma| \rightarrow 0 \\ \sigma_{i}>0 \forall i}} T\left(\frac{b+\sigma}{1+|\sigma|}\right) \leq \lim _{|\sigma| \rightarrow 0} r^{\frac{|\sigma|}{1+|\sigma|}} T^{-}(b)^{\frac{1}{1+|\sigma|}}=T^{-}(b),
$$

which is the desired inequality.

An immediate consequence of Lemma 4.4 and equation (4.4) is the following.

Corollary 4.6. Suppose $T(\phi) \neq 0$ for some $\phi \in \Sigma_{m}^{\circ}$. Then $T(\theta) \neq 0$ for all $\theta \in \Sigma_{m}^{\circ}$ and $T^{-}(b) \neq 0$ for all $b \in \partial \Sigma_{m}$. The same conclusion holds if $T^{-}(c) \neq 0$ for some $c \in \partial \Sigma_{m}$.

Lemma 4.7. Let $Q$ be a compact subset of $\Sigma_{m}^{\circ}$. Then

$$
\limsup _{|\alpha| \rightarrow \infty}\left\{\left|Y(\alpha)^{\frac{1}{|\alpha|}}-T(\theta(\alpha))\right|: \frac{\alpha}{|\alpha|}=: \theta(\alpha) \in Q\right\}=0 .
$$

If $T$ is as in the previous corollary, then also

$$
\limsup _{|\alpha| \rightarrow \infty}\left\{\left|\log Y(\alpha)^{\frac{1}{|\alpha|}}-\log T(\theta(\alpha))\right|: \frac{\alpha}{|\alpha|}=: \theta(\alpha) \in Q\right\}=0 .
$$

Proof. Let $L$ denote the first limsup, and let $\left\{\alpha_{(j)}\right\}$ be a sequence for which

$$
\lim _{j \rightarrow \infty}\left|Y\left(\alpha_{(j)}\right)^{\frac{1}{\alpha_{(j)}}}-T\left(\theta_{(j)}\right)\right|=L
$$

where $\theta_{(j)}=\frac{\alpha_{(j)}}{\left|\alpha_{(j)}\right|}$. We may assume that $\theta_{(j)} \rightarrow \theta \in Q$ by passing perhaps to a subsequence. Then

$$
\left|Y\left(\alpha_{(j)}\right)^{\frac{1}{|\alpha(j)|}}-T\left(\theta_{(j)}\right)\right| \leq\left|Y\left(\alpha_{(j)}\right)^{\frac{1}{|\alpha(j)|}}-T(\theta)\right|+\left|T(\theta)-T\left(\theta_{(j)}\right)\right|
$$

and as $j \rightarrow \infty$, the first expression on the right-hand side goes to zero by Lemma 4.3 and the second by continuity of $T$ (Lemma 4.4). So $L=0$ as required.

If $T$ is as in the previous corollary, then all quantities inside the second limsup are finite. To prove this second statement, one do a similar argument as above, writing $\log Y\left(\alpha_{(j)}\right)^{1 /\left|\alpha_{(j)}\right|}, \log T\left(\theta_{(j)}\right)$, etc. in place of $Y\left(\alpha_{(j)}\right)^{1 /\left|\alpha_{(j)}\right|}, T\left(\theta_{(j)}\right)$.

For a positive integer $s$, let $h_{m}(s)$ denote the number of elements in the set $\left\{\alpha \in \mathbb{Z}_{\geq 0}^{m}:|\alpha|=s\right\} ;$ we have $h_{m}(s)=\left(\begin{array}{c}s+m-1 \\ s\end{array}\right)=\frac{(s+m-1) !}{s !(m-1) !}$.

Lemma 4.8. We have

$$
\frac{1}{h_{m}(s)} \sum_{|\alpha|=s} \log Y(\alpha)^{\frac{1}{|\alpha|}} \longrightarrow \frac{1}{\operatorname{vol}\left(\Sigma_{m}\right)} \int_{\Sigma_{m}^{\circ}} \log T(\theta) d \theta \quad \text { as } s \rightarrow \infty,
$$

where on the right-hand side we integrate over $\theta$ with respect to the usual $m$ dimensional volume on $\mathbb{R}^{m}$, with $\operatorname{vol}\left(\Sigma_{m}\right)=\int_{\Sigma_{m}} d \theta$.

Proof. By Corollary 4.6 we have two cases: either $T$ is never zero on $\Sigma_{m}^{\circ}$ or $T \equiv 0$.

We consider the first case. For convenience write $\theta(\alpha)=\frac{\alpha}{|\alpha|}$. The set $\Sigma(s):=$ $\{\theta(\alpha):|\alpha|=s\}$ is a uniformly distributed grid of points on $\Sigma_{m}$ such that the discrete probability measure $\frac{1}{h_{m}(s)} \sum_{|\alpha|=s} \delta_{\theta(\alpha)}$ supported on $\Sigma(s)$ converges weak* 
to $\frac{1}{\operatorname{vol}\left(\Sigma_{m}\right)} d \theta$ as $s \rightarrow \infty$. Since $\theta \rightarrow T(\theta)$ is a bounded continuous function on $\Sigma_{m}^{\circ}$ and $\operatorname{vol}(\partial \Sigma)=0$,

$$
\frac{1}{h_{m}(s)} \sum_{|\alpha|=s} \log T(\theta(\alpha)) \longrightarrow \frac{1}{\operatorname{vol}\left(\Sigma_{m}\right)} \int_{\Sigma_{m}^{\circ}} \log T(\theta) d \theta \quad \text { as } s \rightarrow \infty .
$$

(To see this, note that the formula holds by weak* convergence when $\log T(\theta)$ is replaced by $(1-\chi) \log T(\theta)$ with $\chi$ an arbitrary smooth cutoff function supported in a neighborhood of $\partial \Sigma$; now shrink the support of $\chi$.)

Hence to prove (4.6), it is sufficient to show that

$$
\left(\frac{1}{h_{m}(s)} \sum_{|\alpha|=s}\left|\log Y(\alpha)^{\frac{1}{|\alpha|}}-\log T(\theta(\alpha))\right|\right) \longrightarrow 0 \quad \text { as } s \rightarrow \infty .
$$

Fix $\delta>0$ and define the compact set $Q_{\delta}:=\left\{\theta=\left(\theta_{1}, \ldots, \theta_{m}\right) \in \Sigma_{m}^{\circ}: \theta_{\nu} \geq \delta \forall \nu\right\}$. For a positive integer $s$, let

$$
L_{1}(s):=\left\{\alpha=\left(\alpha_{1}, \ldots, \alpha_{m}\right) \in \mathbb{Z}_{\geq 0}^{m}:|\alpha|=s, \frac{\alpha}{|\alpha|} \in Q_{\delta}\right\}
$$

and let $L_{2}(s):=\mathbb{Z}_{\geq 0}^{m} \backslash L_{1}(s) ;$ write

$$
L_{2}(s)=\bigcup_{\nu=1}^{m}\left\{\alpha \in L_{2}(s): \frac{\alpha_{\nu}}{s}<\delta\right\}=: \bigcup_{\nu=1}^{m} L_{2, \nu}(s) .
$$

Using $\alpha_{\nu}<\delta s$ and $\sum_{\eta \neq \nu} \alpha_{\eta} \leq s$, we can estimate the size of $L_{2, \nu}(s)$ for each $\nu$ as $\left|L_{2, \nu}(s)\right| \leq \delta s\left(\begin{array}{c}s+m-2 \\ s\end{array}\right)$. A calculation then gives

$$
\frac{\left|L_{2}(s)\right|}{h_{m}(s)}=\sum_{\nu=1}^{m} \frac{\left|L_{2, \nu}(s)\right|}{h_{m}(s)} \leq m \cdot \frac{\delta s\left(\begin{array}{c}
s+m-2 \\
s
\end{array}\right)}{\left(\begin{array}{c}
s+m-1 \\
s
\end{array}\right)} \leq \delta m^{2}
$$

Hence

$$
\begin{aligned}
& \frac{1}{h_{m}(s)} \sum_{|\alpha|=s}\left|\log Y(\alpha)^{\frac{1}{|\alpha|}}-\log T(\theta(\alpha))\right| \\
& =\frac{1}{h_{m}(s)} \sum_{\alpha \in L_{1}(s)}\left|\log Y(\alpha)^{\frac{1}{|\alpha|}}-\log T(\theta(\alpha))\right| \\
& \quad+\frac{1}{h_{m}(s)} \sum_{\alpha \in L_{2}(s)}\left|\log Y(\alpha)^{\frac{1}{|\alpha|}}-\log T(\theta(\alpha))\right| \\
& \leq \frac{\left|L_{1}(s)\right|}{h_{m}(s)} \sup \left\{\left|\log Y(\alpha)^{\frac{1}{|\alpha|}}-\log T(\theta(\alpha))\right|:|\alpha|=s, \theta(\alpha) \in Q_{\delta}\right\} \\
& \quad+\frac{\left|L_{2}(s)\right|}{h_{m}(s)}\left(C^{\frac{1}{s}}+1\right) r \\
& \leq \sup \left\{\left|\log Y(\alpha)^{\frac{1}{|\alpha|}}-\log T(\theta(\alpha))\right|:|\alpha|=s, \theta(\alpha) \in Q_{\delta}\right\}+\delta m^{2}\left(C^{\frac{1}{s}}+1\right) r,
\end{aligned}
$$

with $C, r$ as in Definition 4.1. By Lemma 4.7 the sup in the above line goes to zero as $s \rightarrow \infty$, so

$$
\limsup _{s \rightarrow \infty}\left(\frac{1}{h_{m}(s)} \sum_{|\alpha|=s}\left|\log Y(\alpha)^{\frac{1}{|\alpha|}}-\log T(\theta(\alpha))\right|\right) \leq \delta m^{2} 2 r .
$$

Since $\delta>0$ was arbitrary, (4.7) follows. 
For the case $T \equiv 0$, we need to show that the left-hand side of (4.6) goes to $-\infty$ as $s \rightarrow \infty$. Fix a compact subset $Q$ of $\Sigma_{m}^{\circ}$. The first part of the previous lemma yields

$$
\lim \sup \left\{Y(\alpha)^{1 /|\alpha|}:|\alpha| \rightarrow \infty, \alpha /|\alpha| \in Q\right\}=0 .
$$

Hence given $\epsilon>0$,

$$
\sup \left\{Y(\alpha)^{1 /|\alpha|}:|\alpha|>N, \alpha /|\alpha| \in Q\right\}<\epsilon
$$

for sufficiently large $N$. Using the notation $L_{1}(s), L_{2}(s)$ from the proof of the first case (with $Q$ in place of $Q_{\delta}$ ), we have

$$
\frac{1}{h_{m}(s)} \sum_{|\alpha|=s, \frac{\alpha}{|\alpha|} \in Q} \log Y(\alpha)^{\frac{1}{|\alpha|}} \leq \frac{1}{h_{m}(s)} \sum_{|\alpha|=s, \frac{\alpha}{|\alpha|} \in Q} \log \epsilon=\frac{\left|L_{1}(s)\right|}{h_{m}(s)} \log \epsilon \leq \log \epsilon
$$

for $s>N$. Finally, note that $Y(\alpha)^{1 /|\alpha|}$ is uniformly bounded above for all $\alpha$ (say by some constant $M)$ since $Y$ has subexponential growth. For all $s$,

$$
\frac{1}{h_{m}(s)} \sum_{|\alpha|=s, \frac{\alpha}{|\alpha|} \notin Q} \log Y(\alpha)^{\frac{1}{\alpha \alpha}}=\frac{\left|L_{2}(s)\right|}{h_{m}(s)} M \leq M .
$$

Altogether, $\frac{1}{h_{m}(s)} \sum_{|\alpha|=s} \log Y(\alpha)^{\frac{1}{|\alpha|}} \leq M+\log \epsilon$ when $s>N$. Since $\epsilon$ is arbitrary, the left-hand side of (4.6) goes to $-\infty$ as required.

\section{Chebyshev constants}

In this section we construct Chebyshev constants on an algebraic variety $V \subseteq \mathbb{C}^{n}$. Suppose that $V$ satisfies the properties (3.1). As before, $R:=\mathbb{C}\left[z_{1}, \ldots, z_{m}\right] \subseteq \mathbb{C}[V]$ is a Noether normalization, and $\mathbf{v}_{1}, \ldots, \mathbf{v}_{d}$ are the polynomials of Section 2. We will write $\lambda_{1}, \ldots, \lambda_{d}$ for the interpolating points denoted by $p_{1}, \ldots, p_{d}$ earlier, so that we can use the letter ' $p$ ' to denote polynomials. We also introduce some additional notation.

Notation 5.1. Recall that the basis $\mathcal{C}$ of $\mathbb{C}[V]$ was constructed in Definition 3.13, ordered by $\prec$. Denote by $\left\{\mathbf{e}_{j}\right\}_{j=1}^{\infty}$ the enumeration of $\mathcal{C}$ according to $\prec$. For $f=$ $\sum_{j} a_{j} \mathbf{e}_{j} \in \mathbb{C}[V]$ we write $\operatorname{LT}_{\prec}(f)=a_{k} \mathbf{e}_{k}$ for the leading term, i.e., $a_{k} \neq 0$ and $a_{j}=0$ for all $j>k$. For $f, g \in \mathbb{C}[V]$, write $f \prec g$ if $\operatorname{LT}_{\prec}(f) \prec \operatorname{LT} \prec(g)$.

In what follows, $\alpha$ will always denote a multi-index in $\mathbb{Z}_{\geq 0}^{m}$, and we write $\alpha=$ $\left(\alpha^{\prime}, \alpha_{m}\right)$ where $\alpha^{\prime} \in \mathbb{Z}_{\geq 0}^{m-1}$ and $\alpha_{m} \in \mathbb{Z}_{\geq 0}$. For convenience, we will also identify $\alpha$ and $\alpha^{\prime}$ with $\left(\alpha_{1}, \ldots, \alpha_{m}, 0, \ldots, 0\right)$ and $\left(\alpha_{1}, \ldots, \alpha_{m-1}, 0, \ldots, 0\right)$ in $\mathbb{Z}_{\geq 0}^{n}$ when using multi-index notation (i.e., in expressions such as $\left.z^{\alpha}\right)$.

Definition 5.2. Let $\alpha \in \mathbb{Z}_{\geq 0}^{m}$ be a multi-index. Define for $i=1, \ldots, d$ the collection of polynomials

$$
\mathcal{M}_{i}(\alpha):=\left\{p(z) \in \mathbb{C}[V]: p(z)=z^{\alpha} \mathbf{v}_{i}+g(z), g(z) \prec z^{\alpha} \mathbf{v}_{i}\right\} .
$$

Fix a compact set $K \subseteq V$. We define the function $Y_{i}: \mathbb{Z}_{\geq 0}^{m} \rightarrow \mathbb{R}_{\geq 0}$ by

$$
Y_{i}(\alpha):=\inf \left\{\|p\|_{K}: p \in \mathcal{M}_{i}(\alpha)\right\} .
$$

For a fixed $i \in\{1, \ldots, d\}$, we will write $\ell_{i}\left(z^{\alpha}\right)$ to denote an arbitrary $g \in \mathbb{C}[V]$ with $g \prec z^{\alpha} \mathbf{v}_{i}$. An immediate consequence of Lemma 3.7 is the following. 
Lemma 5.3. We have $\mathbf{v}_{i}^{2}=z_{m}^{t} \mathbf{v}_{i}+\ell_{i}\left(z_{m}^{t}\right)$ and $\mathbf{v}_{i} \mathbf{v}_{j}=\ell_{i}\left(z_{m}^{t}\right)$. Hence if $p \in \mathcal{M}_{i}(\alpha)$, $q \in \mathcal{M}_{i}(\tilde{\alpha})$, then $p q \in \mathcal{M}_{i}\left(\alpha+\tilde{\alpha}+\gamma_{m}\right)$, where $\gamma_{m}=(0, \ldots, 0, t, 0, \ldots, 0)$, where the $t$ is in the $m$-th slot.

Corollary 5.4. The function $Y_{i}$ is weakly submultiplicative with subexponential growth. In particular,

$$
Y_{i}\left(\alpha+\tilde{\alpha}+\gamma_{m}\right) \leq Y_{i}(\alpha) Y_{i}(\tilde{\alpha}), \quad \alpha, \tilde{\alpha} \in \mathbb{Z}_{\geq 0}^{m} .
$$

Proof. Fix indices $\alpha, \tilde{\alpha} \in \mathbb{Z}_{\geq 0}^{m}$. Choose $p \in \mathcal{M}_{i}(\alpha)$ such that $\|p\|_{K}=Y_{i}(\alpha)$ and $q \in \mathcal{M}_{i}(\tilde{\alpha})$ such that $\|q\|_{K}=Y_{i}(\tilde{\alpha})$. By the previous lemma, $p q \in \mathcal{M}_{i}\left(\alpha+\tilde{\alpha}+\gamma_{m}\right)$, so that $Y_{i}\left(\alpha+\tilde{\alpha}+\gamma_{m}\right) \leq\|p q\|_{K} \leq\|p\|_{K}\|q\|_{K}=Y_{i}(\alpha) Y_{i}(\tilde{\alpha})$.

Choose $r$ such that $K \subseteq B(0, r)=\left\{z \in \mathbb{C}^{n}:|z| \leq r\right\}$. Then $Y_{i}(\alpha) \leq r^{|\alpha|}\left\|\mathbf{v}_{i}\right\|_{K}$, so $Y_{i}$ has subexponential growth.

As a consequence of the results in the previous section, we have the following.

Proposition 5.5. The limit

$$
T\left(K, \lambda_{i}, \theta\right):=\lim _{\substack{|\alpha| \rightarrow \infty \\ \alpha /|\alpha| \rightarrow \theta}} Y_{i}(\alpha)^{\frac{1}{|\alpha|}}
$$

exists for each $\theta \in \Sigma_{m}^{\circ}$, and $\theta \mapsto T\left(K, \lambda_{i}, \theta\right)$ defines a logarithmically homogeneous function on $\Sigma_{m}^{\circ}$. Moreover, we have the convergence

$$
\frac{1}{h_{m}(s)} \sum_{|\alpha|=s} \log Y_{i}(\alpha)^{\frac{1}{|\alpha|}} \longrightarrow \frac{1}{\operatorname{vol}\left(\Sigma_{m}\right)} \int_{\Sigma_{m}^{\circ}} \log T\left(K, \lambda_{i}, \theta\right) d \theta \quad \text { as } s \rightarrow \infty .
$$

Definition 5.6. We call $T\left(K, \lambda_{i}, \theta\right)$ the directional Chebyshev constant of $K$ associated to $\lambda_{i}$ and $\theta$.

We call

$$
T\left(K, \lambda_{i}\right):=\exp \left(\frac{1}{\operatorname{vol}\left(\Sigma_{m}\right)} \int_{\Sigma_{m}^{\circ}} \log T\left(K, \lambda_{i}, \theta\right) d \theta\right)
$$

the principal Chebyshev constant of $K$ associated to $\lambda_{i}$.

As in (4.3), we also define $T^{-}\left(K, \lambda_{i}, b\right):=\liminf _{|\alpha| \rightarrow \infty, \frac{\alpha}{|\alpha|} \rightarrow b} Y(\alpha)^{\frac{1}{|\alpha|}}$ for $b \in \partial \Sigma_{m}$.

In the proof of the main theorem on transfinite diameter, we will need to account for polynomials whose leading terms in $\mathcal{C}$ are of the form $(*)$. For $\alpha^{\prime} \in \mathbb{Z}_{\geq 0}^{m-1}$ define

$$
\tilde{\mathcal{M}}\left(\alpha^{\prime}\right):=\left\{p \in \mathbb{C}[V]: \mathrm{LT}_{\prec}(p)=z^{\alpha^{\prime}} z_{m}^{l} z^{\beta} \text { with } z_{m}^{l} z^{\beta} \in \mathcal{B}\right\} .
$$

Recall that this means that $l+|\beta|<t$. Set $\tilde{Y}\left(\alpha^{\prime}\right):=\inf \left\{\|p\|_{K}: p \in \tilde{\mathcal{M}}\left(\alpha^{\prime}\right)\right\}$. If $K \subseteq B(0, r)$ it is easy to see that

$$
\tilde{Y}\left(\alpha^{\prime}\right) \leq r^{\left|\alpha^{\prime}\right|} .
$$

Also, set $\tilde{T}\left(\alpha^{\prime}\right):=\inf \left\{\|p\|_{K}^{\frac{1}{\operatorname{deg} p}}: p \in \tilde{\mathcal{M}}\left(\alpha^{\prime}\right)\right\}$ and define the function

$$
\tilde{T}^{-}\left(K, \theta^{\prime}\right):=\liminf _{\left|\alpha^{\prime}\right| \rightarrow \infty, \frac{\alpha^{\prime}}{\left|\alpha^{\prime}\right|} \rightarrow \theta^{\prime}} \tilde{T}\left(\alpha^{\prime}\right)
$$

on $\Sigma_{m-1}:=\left\{\theta^{\prime}=\left(\theta_{1}, \ldots, \theta_{m-1}\right) \in \mathbb{R}^{m-1}: \sum_{k} \theta_{k}=1\right\}$. We want to get a lower estimate for this quantity. First we make the following observation. Since the 
monomial $z_{m}^{t-|\beta|} z^{\beta}$ is not in $\mathcal{B}$ it must be expressed in $\mathbb{C}[V]$ with respect to the basis $\mathcal{C}$ as

$$
z_{m}^{t-|\beta|} z^{\beta}=\sum_{i=1}^{d} C_{\beta i} \mathbf{v}_{i}+q(z)
$$

where $\operatorname{deg} q \leq t, \mathrm{LT}_{\prec}(q) \prec \mathbf{v}_{1}$, and not all $C_{\beta i}$ are zero.

Lemma 5.7. Suppose $C_{l \beta i} \neq 0$ for some $i \in\{1, \ldots, d\}$. Then for each $\theta^{\prime} \in \Sigma_{m-1}$ we have

$$
T^{-}\left(K, \lambda_{i}, \theta\right) \leq \tilde{T}^{-}\left(K, \theta^{\prime}\right),
$$

where $\theta=\left(\theta^{\prime}, 0\right)=\left(\theta_{1}, \ldots, \theta_{m-1}, 0\right) \in \partial \Sigma_{m}$.

Proof. Fix $\theta^{\prime} \in \Sigma_{m-1}$ and let $\epsilon>0$. Let $\left\{\alpha_{(j)}^{\prime}\right\}$ be a sequence in $\mathbb{Z}_{\geq 0}^{m-1}$ with $\left|\alpha_{(j)}^{\prime}\right| \rightarrow \infty, \frac{\alpha_{(j)}^{\prime}}{\left|\alpha_{(j)}^{\prime}\right|} \rightarrow \theta^{\prime}$, and $\tilde{T}\left(\alpha_{(j)}^{\prime}\right) \rightarrow \tilde{T}^{-}\left(K, \theta^{\prime}\right)$ as $j \rightarrow \infty$.

Next, choose a sequence of polynomials $\left\{p_{j}\right\} \subseteq \mathbb{C}[V]$ such that $p_{j} \in \tilde{\mathcal{M}}\left(\alpha_{(j)}^{\prime}\right)$ and $\|p\|_{K}^{1 / \operatorname{deg} p_{j}} \leq \tilde{T}\left(\alpha_{(j)}^{\prime}\right)+\epsilon$. Since $\mathcal{B}$ is finite, we can assume, by passing perhaps to a subsequence, that $\operatorname{LT}_{\prec}\left(p_{j}\right)=z^{\alpha_{(j)}^{\prime}} z_{m}^{l} z^{\beta}$ where $l$ and $\beta$ are the same for all $j$.

Let $Q:=C_{\beta i}^{-1} z_{m}^{t-l-|\beta|} \mathbf{v}_{i}$ and define $\left\{P_{j}\right\} \subseteq \mathbb{C}[V]$ by $P_{j}:=Q p_{j}$ for each $j$. Then a calculation using equation (5.2) and Lemma 5.3 shows that $P_{j} \in \mathcal{M}_{i}\left(\alpha_{(j)}\right)$ where $\alpha_{(j)}=\left(\alpha_{(j)}^{\prime}, t-|\beta|\right)$. Clearly $\frac{\alpha_{(j)}}{\left|\alpha_{(j)}\right|} \rightarrow \theta$ as $j \rightarrow \infty$ since $l$ and $|\beta|$ are bounded from above by $t$. Now

$$
Y_{i}\left(\alpha_{(j)}\right)^{\frac{1}{\alpha(j)}} \leq\|Q\|_{K}^{\frac{1}{\alpha \alpha_{(j)}}}\left\|p_{j}\right\|_{K}^{\frac{1}{\alpha_{(j)}}} \leq\|Q\|_{K}^{\frac{1}{\alpha \alpha}(j)}\left(\tilde{T}\left(\alpha_{(j)}^{\prime}\right)+\epsilon\right)^{\frac{\operatorname{deg} p_{j}}{\lceil\alpha(j)}} .
$$

We take the $\lim \inf$ as $j \rightarrow \infty$. We have $T^{-}\left(K, \lambda_{i}, \theta\right) \leq \tilde{T}^{-}\left(K, \theta^{\prime}\right)+\epsilon$ since $\frac{\operatorname{deg} p_{j}}{\left|\alpha_{(j)}\right|} \rightarrow 1$, and (5.3) follows since $\epsilon$ was arbitrary.

Corollary 5.8. We have

$\liminf _{\left|\alpha^{\prime}\right| \rightarrow \infty} \tilde{Y}\left(\alpha^{\prime}\right)^{\frac{1}{\left|\alpha^{\prime}\right|}}=\liminf _{\left|\alpha^{\prime}\right| \rightarrow \infty} \tilde{T}(\alpha) \geq \min \left\{T^{-}\left(K, \lambda_{i}, \theta\right): i \in\{1, \ldots, d\}, \theta \in \partial \Sigma_{m}\right\}$.

\section{The transfinite Diameter}

Recall that $\left\{\mathbf{e}_{j}\right\}_{j=1}^{\infty}$ denotes the enumeration of the basis $\mathcal{C}$ according to the ordering $\prec$. For a finite set $\left\{\zeta_{1}, \ldots, \zeta_{s}\right\} \subseteq V$, define

$$
\operatorname{Van}_{\mathcal{C}}\left(\zeta_{1}, \ldots, \zeta_{s}\right):=\operatorname{det}\left(\begin{array}{cccc}
1 & 1 & \cdots & 1 \\
\mathbf{e}_{2}\left(\zeta_{1}\right) & \mathbf{e}_{2}\left(\zeta_{2}\right) & \cdots & \mathbf{e}_{2}\left(\zeta_{s}\right) \\
\vdots & \vdots & \ddots & \vdots \\
\mathbf{e}_{s}\left(\zeta_{1}\right) & \mathbf{e}_{s}\left(\zeta_{2}\right) & \cdots & \mathbf{e}_{s}\left(\zeta_{s}\right)
\end{array}\right)
$$

As in the previous section, fix a compact set $K \subseteq V$. We have $K \subseteq B(0, r)=$ $\{|z|<r\}$ for some $r>0$. 
Notation 6.1. For a positive integer $s$,

$$
V_{s}:=\sup \left\{\left|\operatorname{Van}_{\mathcal{C}}\left(\zeta_{1}, \ldots, \zeta_{s}\right)\right|:\left\{\zeta_{1}, \ldots, \zeta_{s}\right\} \subseteq K\right\} .
$$

Also, given any positive integer $s$, let $h_{s}$ denote the dimension of $\mathbb{C}[V]_{=s}$, let $m_{s}:=$ $\sum_{\nu=0}^{s} h_{\nu}$ denote the dimension of $\mathbb{C}[V]_{\leq s}$, and let $l_{s}:=\sum_{\nu=0}^{s} \nu h_{\nu}$ denote the sum of the degrees of the basis elements $\mathcal{C} \cap \mathbb{C}[V]_{\leq s}$.

We now state our main theorem.

Theorem 6.2. The limit $d(K)=\lim _{s \rightarrow \infty} V_{m_{s}}^{1 / l_{s}}$ exists and we have the formula

$$
d(K)=\left(\prod_{i=1}^{d} T\left(K, \lambda_{i}\right)\right)^{1 / d} .
$$

To prove the theorem we will need some lemmas. Recall that $\mathcal{B}$ is the collection of monomials given by (3.7).

Lemma 6.3. Let $s$ be a positive integer. If $\mathbf{e}_{s}=z^{\alpha} \mathbf{v}_{i}$ for some integer $i \in\{1, \ldots, d\}$ then

$$
Y_{i}(\alpha) \leq \frac{V_{s}}{V_{s-1}} \leq s Y_{i}(\alpha) .
$$

If $\mathbf{e}_{s}=z^{\alpha^{\prime}} z_{m}^{l} z^{\beta}$ with $z_{m}^{l} z^{\beta} \in \mathcal{B} \cap \mathcal{C}$, then

$$
\tilde{Y}\left(\alpha^{\prime}\right) \leq \frac{V_{s}}{V_{s-1}} \leq s \tilde{Y}_{i}\left(\alpha^{\prime}\right)
$$

Proof. Choose points $\zeta_{1}, \ldots, \zeta_{s-1}$ in $K$ such that $\operatorname{Van}_{\mathcal{C}}\left(\zeta_{1}, \ldots, \zeta_{s-1}\right)=V_{i-1}$. It is easy to see that the polynomial $P(z):=\frac{\operatorname{Van}_{\mathcal{C}}\left(\zeta_{1}, \ldots, \zeta_{s-1}, z\right)}{\operatorname{Van}_{\mathcal{C}}\left(\zeta_{1}, \ldots, \zeta_{s-1}\right)}$ is in $\mathcal{M}(\alpha)$ by expanding the determinant, and hence

$$
Y_{i}(\alpha) \leq\|P\|_{K} \leq \frac{V_{s}}{V_{s-1}}
$$

which gives the first inequality of (6.2).

Now choose points $\zeta_{1}, \ldots, \zeta_{s}$ in $K$ such that $\operatorname{Van}_{\mathcal{C}}\left(\zeta_{1}, \ldots, \zeta_{s}\right)=V_{i}$ and let $t(z)=\mathbf{e}_{s}+\sum_{\nu<s} c_{\nu} \mathbf{e}_{\nu}$ be a polynomial in $\mathcal{M}(\alpha)$ such that $\|t\|_{K}=Y_{i}(\alpha)$. Then by properties of determinants,

$$
\begin{aligned}
V_{i} & =\left|\operatorname{det}\left(\begin{array}{cccc}
1 & 1 & \cdots & 1 \\
\mathbf{e}_{2}\left(\zeta_{1}\right) & \mathbf{e}_{2}\left(\zeta_{2}\right) & \cdots & \mathbf{e}_{2}\left(\zeta_{s}\right) \\
\vdots & \vdots & \ddots & \vdots \\
\mathbf{e}_{s-1}\left(\zeta_{1}\right) & \mathbf{e}_{s-1}\left(\zeta_{2}\right) & \cdots & \mathbf{e}_{s-1}\left(\zeta_{s}\right) \\
t\left(\zeta_{1}\right) & t\left(\zeta_{2}\right) & \cdots & t\left(\zeta_{s}\right)
\end{array}\right)\right| \\
& \leq \sum_{\nu=1}^{s}\left|t\left(\zeta_{\nu}\right)\right|\left|V\left(\zeta_{1}, \ldots, \widehat{\zeta}_{\nu}, \ldots, \zeta_{s}\right)\right| \leq \sum_{\nu=1}^{s} Y_{i}(\alpha) V_{s-1}=s Y_{i}(\alpha) V_{s-1},
\end{aligned}
$$

where we expand along the bottom row. This gives the second inequality of (6.2).

The proof of (6.3) is similar, so we omit it.

We need to keep track of exponents. Let $t$ be as in Section 3 (see the paragraph following Corollary 3.3). Fix an integer $s>t$. For an element $z^{\alpha} \mathbf{v}_{i}$ there are $d$ 
choices for $i$ and $h_{m}(s-t)=\left(\begin{array}{c}s-t+m-1 \\ m-1\end{array}\right)=\frac{(s-t+m-1) !}{(s-t) !(m-1) !}$ choices for $\alpha$ when $|\alpha|=s-t$. Hence the number of basis elements of degree $s$ of the form $(* *)$ is $d h_{m}(s-t)$.

Let $a_{s}:=h_{s}-d h_{m}(s-t)$ be the number of remaining basis elements, of the form (*), i.e., $z^{\alpha^{\prime}} a z_{m}^{l} z^{\beta}$ with $\alpha^{\prime} \in \mathbb{Z}_{\geq 0}^{m-1}$ and $z_{m}^{l} z^{\beta} \in \mathcal{B}$. We then have the estimate $a_{s} \leq|\mathcal{B}|\left(\begin{array}{c}s+m-2 \\ m-2\end{array}\right)$, where $|\mathcal{B}|$ denotes the size of the set $\mathcal{B}$. Hence

$$
\frac{a_{s}}{h_{s}} \leq \frac{|\mathcal{B}|\left(\begin{array}{c}
s+m-2 \\
m-2
\end{array}\right)}{d\left(\begin{array}{c}
s-t+m-1 \\
m-1
\end{array}\right)} \longrightarrow 0 \quad \text { as } s \rightarrow \infty, \quad \text { and so } \frac{d h_{m}(s-t)}{h_{s}} \longrightarrow 1 .
$$

Let $\tilde{T}_{s}:=\inf \left\{\tilde{T}\left(\alpha^{\prime}\right): s-t \leq\left|\alpha^{\prime}\right| \leq s\right\}$. A straightforward corollary of the previous lemma is the following.

Corollary 6.4. For a positive integer $s>t$, we have

$$
\tilde{T}_{s}^{s a_{s}}\left(\prod_{|\alpha|=s} \prod_{i=1}^{d} Y_{i}(\alpha)\right) \leq \frac{V_{m_{s}}}{V_{m_{s-1}}} \leq\left(\frac{m_{s} !}{m_{s-1} !}\right)^{2} r^{s a_{s}} \prod_{|\alpha|=s} \prod_{i=1}^{d} Y_{i}(\alpha) .
$$

Proof. We apply Lemma 6.3 to the product $\frac{V_{m_{s}}}{V_{m_{s-1}}}=\frac{V_{m_{s}}}{V_{m_{s}-1}} \frac{V_{m_{s}-1}}{V_{m_{s}-2}} \cdots \frac{V_{m_{s-1}+1}}{V_{m_{s-1}}}$. For the upper estimate, we have

$$
\begin{aligned}
\frac{V_{m_{s}}}{V_{m_{s-1}}}= & \frac{V_{m_{s}}}{V_{m_{s}-1}} \frac{V_{m_{s}-1}}{V_{m_{s}-2}} \cdots \frac{V_{m_{s-1}+1}}{V_{m_{s-1}}} \\
= & \left(\frac{V_{m_{s}}}{V_{m_{s}-1}} \cdots \frac{V_{m_{s-1}+a_{s}+1}}{V_{m_{s-1}+a_{s}}}\right)\left(\frac{V_{m_{s-1}+a_{s}}}{V_{m_{s-1}+a_{s}-1}} \cdots \frac{V_{m_{s-1}+1}}{V_{m_{s-1}}}\right) \\
\leq & \left(m_{s} m_{s-1} \cdots\left(m_{s-1}+a_{s}+1\right) \prod_{|\alpha|=s} \prod_{i=1}^{d} Y_{i}(\alpha)\right) \\
& \quad \times\left(\left(m_{s-1}+a_{s}\right) \cdots\left(m_{s-1}+1\right) \prod_{\nu=m_{s-1}+1}^{m_{s-1}+a_{s}} \tilde{Y}\left(\alpha^{\prime}\left(\mathbf{e}_{\nu}\right)\right)\right),
\end{aligned}
$$

where in the last two lines the first large parentheses applies (6.2) to those fractions $V_{k} / V_{k-1}$ for which $\mathbf{e}_{\nu}$ is of the form $(* *)$ while the second large parentheses applies (6.3) to those fractions for which $\mathbf{e}_{\nu}$ is of the form $(*)$. We have also written $\alpha^{\prime}\left(\mathbf{e}_{\nu}\right)$ to denote the multi-index $\alpha^{\prime} \in \mathbb{Z}_{\geq 0}^{m-1}$ for which $\mathbf{e}_{\nu}=z^{\alpha^{\prime}} z_{m}^{l} z^{\beta}$. We have

$$
\begin{aligned}
\left(m_{s} m_{s-1} \cdots(\right. & \left.\left.m_{s-1}+a_{s}+1\right) \prod_{|\alpha|=s} \prod_{i=1}^{d} Y_{i}(\alpha)\right) \\
& \times\left(\left(m_{s-1}+a_{s}\right) \cdots\left(m_{s-1}+1\right) \prod_{\nu=m_{s-1}+1}^{m_{s-1}+a_{s}} \tilde{Y}\left(\alpha^{\prime}\left(\mathbf{e}_{\nu}\right)\right)\right) \\
\leq & \left(\frac{m_{s} !}{m_{s-1} !} \prod_{|\alpha|=s} \prod_{i=1}^{d} Y_{i}(\alpha)\right)\left(\frac{m_{s} !}{m_{s-1} !} \prod_{\nu=m_{s-1}+1}^{m_{s-1}+a_{s}} r^{s}\right)
\end{aligned}
$$

where we use (5.1) in the last line. This last expression is the upper estimate in (6.5). The lower estimate follows similarly, using the fact that $s-t \leq\left|\alpha^{\prime}\left(\mathbf{e}_{\nu}\right)\right| \leq s$ for all $\nu=m_{s-1}+1, \ldots, m_{s-1}+a_{s}$, so that $\tilde{Y}\left(\alpha^{\prime}\left(\mathbf{e}_{\nu}\right)\right) \geq \tilde{T}_{s}^{s}$ for all $\nu$. 
Similar reasoning as in the paragraphs before the above corollary give

$$
m_{s} \leq d\left(\begin{array}{c}
s-t+m \\
m
\end{array}\right)+|\mathcal{B}|\left(\begin{array}{c}
s-t+m-1 \\
m-1
\end{array}\right)
$$

and when $s>t$,

$$
l_{s}=\sum_{\nu=1}^{s} \nu h_{\nu} \geq \sum_{\nu=t}^{s} \nu h_{\nu} \geq \sum_{\nu=1}^{s-t} \nu h_{\nu+t} \geq \sum_{\nu=1}^{s-t} \nu \cdot d\left(\begin{array}{c}
\nu+m-1 \\
m-1
\end{array}\right)=d m\left(\begin{array}{c}
s-t+m \\
m+1
\end{array}\right) .
$$

Then $\frac{m_{s}}{l_{s}} \leq \frac{m+1}{m(s-t)}+\frac{|\mathcal{B}|(m+1)}{d(s-t)(s-t+m)}$, in particular $\frac{m_{s}}{l_{s}} \rightarrow 0$, and

$$
1 \leq\left(m_{s} !\right)^{\frac{1}{l_{s}}} \leq m_{s}^{\frac{m_{s}}{l_{s}}} \longrightarrow 1 \text { as } s \rightarrow \infty \text {. }
$$

Set $T_{s}\left(\lambda_{i}\right):=\left(\prod_{|\alpha|=s-t} Y_{i}(\alpha)\right)^{\frac{1}{s h_{s}}} ;$ then $(\underline{6.5}$ becomes

$$
\tilde{T}_{s}^{s a_{s}} \prod_{i=1}^{d} T_{s}\left(\lambda_{i}\right)^{s h_{s}} \leq \frac{V_{m_{s}}}{V_{m_{s-1}}} \leq r^{s a_{s}}\left(\frac{m_{s} !}{m_{s-1} !}\right)^{2} \prod_{i=1}^{d} T_{s}\left(\lambda_{i}\right)^{s h_{s}}
$$

Write $V_{m_{s}}=\frac{V_{m_{s}}}{V_{m_{s}-1}} \cdots \frac{V_{m_{t+1}}}{V_{m_{t}}} V_{m_{t}}$. Then the above calculation yields the following.

\section{Corollary 6.5.}

$$
\prod_{\nu=t+1}^{s}\left(\tilde{T}_{\nu}^{\nu a_{\nu}} \prod_{i=1}^{d} T_{\nu}\left(\lambda_{i}\right)^{\nu h_{\nu}}\right) V_{m_{t}} \leq V_{m_{s}} \leq\left(m_{s} !\right)^{2} \prod_{\nu=t+1}^{s}\left(r^{\nu a_{\nu}} \prod_{i=1}^{d} T_{\nu}\left(\lambda_{i}\right)^{\nu h_{\nu}}\right) V_{m_{t}} .
$$

To prove Theorem 6.2 we take $l_{s}$-th roots in the above inequality and show that the upper and lower estimates have the desired limit as $s \rightarrow \infty$.

Lemma 6.6. As $s \rightarrow \infty$, we have

$$
\left(m_{s} !\right)^{\frac{2}{l_{s}}} \longrightarrow 1, \quad \frac{\sum_{\nu=t+1}^{s} \nu a_{\nu}}{l_{s}} \longrightarrow 0, \quad \text { and } \frac{s h_{s}}{(s-t) h_{m}(s-t)} \longrightarrow d .
$$

Proof. The first limit follows immediately from (6.6). Writing the left-hand side of the second limit as $\frac{\sum_{\nu=t+1}^{s} \nu a_{\nu}}{\sum_{\nu=1}^{s} \nu h_{\nu}}$, convergence of this limit to zero follows easily from $\frac{a_{s}}{h_{s}} \rightarrow 0$ (the first limit in (6.4) ). The third limit (to $d$ ) follows easily from the second limit in (6.4).

Proof of Theorem 6.2. We first verify that

$$
T_{s}\left(\lambda_{i}\right) \rightarrow T\left(K, \lambda_{i}\right)^{\frac{1}{d}} \quad \text { as } s \rightarrow \infty .
$$

By Proposition 5.5.

$$
\begin{aligned}
\left(\prod_{|\alpha|=s-t} Y_{i}(\alpha)\right)^{\frac{1}{(s-t) h_{m}(s-t)}} & =\exp \left(\frac{1}{h_{m}(s-t)} \sum_{|\alpha|=s-t} \log Y_{i}(\alpha)^{\frac{1}{|\alpha|}}\right) \\
& \longrightarrow T\left(K, \lambda_{i}\right) .
\end{aligned}
$$


Together with the third limit of (6.8) and the definition of $T_{s}\left(\lambda_{i}\right)$, we get (6.9). In turn, writing $\tilde{l}_{s}=\sum_{\nu=t+1}^{s} \nu h_{\nu}$, this gives the convergence

$$
\left(\prod_{\nu=t+1}^{s} T_{\nu}\left(\lambda_{i}\right)^{\nu h_{\nu}}\right)^{1 / \tilde{l}_{s}} \longrightarrow T\left(K, \lambda_{i}\right)^{\frac{1}{d}} \quad \text { as } s \rightarrow \infty
$$

of weighted geometric means. Note that $\tilde{l}_{s} / l_{s} \rightarrow 1$ as $s \rightarrow \infty$, so we may replace $\tilde{l}_{s}$-th roots with $l_{s}$-th roots in what follows. We have

$$
\begin{aligned}
\left(m_{s} !\right)^{\frac{2}{l_{s}}} & \prod_{\nu=t+1}^{s}\left(r^{\nu a_{\nu}} \prod_{i=1}^{d} T_{\nu}\left(\lambda_{i}\right)^{\nu h_{\nu}}\right)^{\frac{1}{l_{s}}} V_{m_{t}}^{\frac{1}{l_{s}}} \\
& =\left(m_{s} !\right)^{\frac{2}{l_{s}}} r^{\frac{\sum \nu a_{\nu}}{l_{s}}} \prod_{i=1}^{d}\left(\prod_{\nu=t+1}^{s} T_{\nu}\left(\lambda_{i}\right)^{\nu h_{\nu}}\right)^{1 / l_{s}} V_{m_{t}}^{\frac{1}{l_{s}}} \longrightarrow\left(\prod_{i=1}^{d} T\left(K, \lambda_{i}\right)\right)^{\frac{1}{d}}
\end{aligned}
$$

as $s \rightarrow \infty$, which shows that $\limsup _{s \rightarrow \infty} V_{m_{s}}^{1 / l_{s}} \leq\left(\prod_{i=1}^{d} T\left(K, \lambda_{i}\right)\right)^{\frac{1}{d}}$.

If $T\left(K, \lambda_{i}\right)=0$ for some $i$ then the theorem is proved, with $d(K)=0$. Otherwise, $T\left(K, \lambda_{i}\right)>0$ for all $i$; using Corollary 4.6 it is easy to see that $T^{-}\left(K, \lambda_{i}, b\right)>0$ for all $i=1, \ldots, d$ and $b \in \partial \Sigma_{m}$; and since $\partial \Sigma_{m}$ is compact, there exists $c>0$ such that $T^{-}\left(K, \lambda_{i}, b\right) \geq c$ for all $i$ and $b$. By Lemma 5.7 .

$$
\liminf _{s \rightarrow \infty} \tilde{T}_{s} \geq \liminf _{\left|\alpha^{\prime}\right| \rightarrow \infty} \tilde{T}\left(\alpha^{\prime}\right) \geq \min _{\theta^{\prime} \in \Sigma_{m-1}} \tilde{T}^{-}\left(\theta^{\prime}\right) \geq \min _{i, b} T^{-}\left(K, \lambda_{i}, b\right) \geq c,
$$

so there is some uniform constant $\epsilon \in(0, c)$ such that $T_{s}>\epsilon$ for all $s>t$, which gives

$$
\prod_{\nu=t+1}^{s}\left(\epsilon^{\nu a_{\nu}} \prod_{i=1}^{d} T_{\nu}\left(\lambda_{i}\right)^{\nu h_{\nu}}\right) V_{m_{t}} \leq V_{m_{s}}
$$

Now the $l_{s}$-th root of the left-hand side of the above goes to $\left(\prod_{i=1}^{d} T\left(K, \lambda_{i}\right)\right)^{\frac{1}{d}}$ as $s \rightarrow \infty$ by a similar argument as before. This concludes the proof.

\section{TRANSFINITE DIAMETER USING THE STANDARD BASIS}

In this section we verify that the transfinite diameter of the previous section may be computed in terms of the standard (grevlex) basis of monomials in $\mathbb{C}[V]$. Recall that the basis for normal forms $\mathbb{C}[z]_{I}$ (where $I=\mathbf{I}(V)$ ) is given by the collection of monomials

$$
\left\{z^{\gamma}: \gamma \in \mathbb{Z}_{\geq 0}, z^{\gamma} \notin\langle\mathrm{LT}(I)\rangle\right\} .
$$

Writing $\left\{\tilde{\mathbf{e}}_{j}\right\}_{j=1}^{\infty}$ for the enumeration of these monomials according to grevlex, define $\operatorname{Van}\left(\zeta_{1}, \ldots, \zeta_{M}\right)$ as in the right-hand side of (6.1) for a finite set $\left\{\zeta_{1}, \ldots, \zeta_{M}\right\} \subseteq V$, replacing $\mathbf{e}_{j}$ 's with $\tilde{\mathbf{e}}_{j}$ 's. Put

$$
W_{m_{s}}:=\sup \left\{\left|\operatorname{Van}\left(\zeta_{1}, \ldots, \zeta_{m_{s}}\right)\right|:\left\{\zeta_{1}, \ldots, \zeta_{m_{s}}\right\} \subseteq K\right\}
$$

Later in this section we will need to consider Vandermonde determinants formed from other graded polynomial bases. The Vandermonde determinant associated to a basis $\mathcal{F}$ will be denoted $\operatorname{Van}_{\mathcal{F}}(\cdot)$. 
Lemma 7.1. Let $\mathcal{F}_{1}=\left\{\tilde{\mathbf{f}}_{j}\right\}_{j=1}^{\infty}$ and $\mathcal{F}_{2}=\left\{\mathbf{f}_{j}\right\}_{j=1}^{\infty}$ be bases of polynomials for $\mathbb{C}[V]$, enumerated according to a graded ordering, and suppose that for some positive integer $M, \tilde{\mathbf{f}}_{\tau}=\mathbf{f}_{\tau}$ whenever $\tau>M$. Then there exists a uniform constant $\kappa \neq 0$ such that for any integer $\tau \geq M$ and finite set $\left\{\zeta_{1}, \ldots, \zeta_{\tau}\right\}$,

$$
\operatorname{Van}_{\mathcal{F}_{1}}\left(\zeta_{1}, \ldots, \zeta_{\tau}\right)=\kappa \operatorname{Van}_{\mathcal{F}_{2}}\left(\zeta_{1}, \ldots, \zeta_{\tau}\right)
$$

Proof. Fix the set $\left\{\zeta_{1}, \ldots, \zeta_{\tau}\right\}$ where $\tau \geq M$. Let $E_{l}=\left[\tilde{\mathbf{f}}_{j}\left(\zeta_{k}\right)\right]_{j, k=1}^{l}$ and $F_{l}=$ $\left[\mathbf{f}_{j}\left(\zeta_{k}\right)\right]_{j, k=1}^{l}$ denote the Vandermonde matrices at the $l$-th stage for $l=1, \ldots, \tau$. With this notation, we have $E_{M}=P_{M} F_{M}$, where $P_{M}$ is the change of basis matrix from $\left\{\tilde{\mathbf{f}}_{j}\right\}_{j=1}^{M}$ to $\left\{\mathbf{f}_{j}\right\}_{j=1}^{M}$ over the linear space spanned by these polynomials. In particular, $\operatorname{det} P_{M} \neq 0$. Taking determinants, $\operatorname{Van}_{\mathcal{F}_{1}}\left(\zeta_{1}, \ldots, \zeta_{M}\right)=$ $\operatorname{det}\left(P_{M}\right) \operatorname{Van}_{\mathcal{F}_{2}}\left(\zeta_{1}, \ldots, \zeta_{M}\right)$.

Similarly, write $E_{\tau}=P_{\tau} F_{\tau}$; then $E_{\tau}$ and $F_{\tau}$ are of the form

$$
E_{\tau}=\left[\frac{E_{M} \mid *}{E^{\prime}}\right], \quad F_{\tau}=\left[\frac{F_{M} \mid *}{E^{\prime}}\right],
$$

the last rows (denoted by $E^{\prime}$ ) being the same since $\mathbf{e}_{l}=\mathbf{f}_{l}$ when $l>M$. It follows that $P_{\tau}$ must be of the form $P_{\tau}=\left[\begin{array}{c|c}P_{M} & * \\ \hline 0 & I\end{array}\right]$ where $I$ denotes the identity matrix, so that $\operatorname{det} P_{\tau}=\operatorname{det} P_{M}$.

Taking $\kappa:=\operatorname{det} P_{M}$, the lemma follows immediately.

Recall that the basis $\mathcal{C}$ of Definition 3.13 is made up of the normal forms of two types of polynomials

$$
\begin{array}{rll}
(*) & z^{\alpha} z_{m}^{l} z^{\beta}: & \alpha \in \mathbb{Z}_{\geq 0}^{m-1}, l+|\beta| \leq t-1 \\
(* *) & z^{\alpha} z_{m}^{l} \mathbf{v}_{i}: & \alpha \in \mathbb{Z}_{\geq 0}^{m-1}, l \geq 0, i=1, \ldots, d .
\end{array}
$$

When these polynomials are already normal forms, as in the examples of Section 3 . we have the following theorem.

Theorem 7.2. Suppose the polynomials $(*)$ and $(* *)$ are already in normal form. Then $\lim _{s \rightarrow \infty} W_{m_{s}}^{1 / l_{s}}=d(K)$. (Here $l_{s}, m_{s}$ are as in Notation 6.1.)

The idea is to show that $\left(V_{m_{s}}^{1 / l_{s}} / W_{m_{s}}^{1 / l_{s}}\right) \rightarrow 1$ as $s \rightarrow \infty$, where $V_{m_{s}}$ is as in the notation of the previous section. To this end, we analyze the Vandermonde determinants that give these quantities in more detail.

Write

$$
\mathbf{v}_{j}(z)=\sum_{\beta \in \mathcal{D}} A_{j \beta} z^{\beta}, \quad j=1, \ldots, d
$$

where $\mathcal{D}$ is the collection of all basis monomials that appear in the polynomials $\mathbf{v}_{j}$ for all $j=1 \ldots, d$. Choose constants $c, C>0$ such that for any positive integer $k \leq d$,

$$
c \leq|\operatorname{det} A| \leq C
$$

whenever $A$ is a $k \times k$ nonsingular square matrix obtained by deleting sufficiently many rows and columns of the $d \times|\mathcal{D}|$ matrix $\left[A_{j \beta}\right]_{j, \beta}$ 团 There are finitely many

\footnotetext{
${ }^{\dagger}$ cf. Remark 3.10

${ }^{\ddagger}$ Since only the absolute value of the determinant appears, the order of the columns (indexed by $\beta$ ) is not important.
} 
possible values for $|\operatorname{det} A|$, so we may take the maximum and minimum of these as our constants.

We are interested in $\left|\operatorname{Van}\left(\zeta_{1}, \ldots, \zeta_{m_{\tau}}\right)\right|$ for a finite set $\left\{\zeta_{1}, \ldots, \zeta_{m_{\tau}}\right\}$. The value is the same for any graded ordering of the monomials of $\mathbb{C}[V]_{<\tau}$, so let us construct yet another graded ordering that will be convenient for calculation.

Fix the usual grevlex ordering on monomials of degree $<t$. For $\tau \geq t$, and supposing that monomials of degree $<\tau$ have already been ordered, we order the monomials of degree $\tau$ as follows. First, list the monomials of the form $(*)$ according to the ordering on $\mathcal{C}$. We set up some convenient notation before continuing.

Notation 7.3. Let $\mathcal{W}_{0}$ be the set consisting of the monomial basis of $\mathbb{C}[V]_{<\tau-1}$ together with the monomials of the form $(*)$ of degree $\tau$. Let $\mathbf{W}_{0}$ denote this same set with our ordering imposed. (With this notation, the matrices given below are uniquely determined.) Also, $\mathbf{W}_{k}$ will have the same meaning when $\mathcal{W}_{k}, k=1,2, \ldots$ is defined later in the section.

Having listed the monomials in $\mathcal{W}_{0}$, we will use the elements of $(* *)$ to order the remaining monomials in $\mathbb{C}[V]_{\leq \tau}$. Before we do this, observe that for $\alpha \in \mathbb{Z}_{\geq 0}^{m}$,

$$
z^{\alpha} \mathbf{v}_{j}=\sum_{\beta \in \mathcal{D}} A_{j \beta} z^{\alpha+\beta}
$$

and since $z^{\alpha} \mathbf{v}_{j}$ is a normal form, each of the monomials in the sum on the right-hand side is a basis monomial.

Returning to the construction of our ordering, let us enumerate the multi-indices $\alpha \in \mathbb{Z}_{\geq 0}^{m}$ of total degree $\tau-t$ as $\alpha(1), \alpha(2), \ldots$, according to their order of appearance in the elements of the form $(* *)$ in $\mathcal{C}$.

The polynomials $\left\{z^{\alpha(1)} \mathbf{v}_{j}\right\}_{j=1}^{d}$ are linearly independent by Theorem 3.12, This allows us to choose, for each $j=1, \ldots, d$, a term $z^{\beta(j)}$ of $z^{\alpha(1)} \mathbf{v}_{j}$ that is not a term of $z^{\alpha(1)} \mathbf{v}_{i}$ whenever $i<j$. We can also arrange that none of these terms be in $\mathcal{W}_{0}$ either, since by the construction of $\mathcal{C}$ in Section 2 , none of the polynomials $z^{\alpha(1)} \mathbf{v}_{j}$ are in the span of $\mathcal{W}_{0}$. The set of monomials defined by

$$
\mathcal{W}_{1}:=\left\{z^{\gamma}: z^{\gamma} \in \mathcal{W}_{0} \text { or } z^{\gamma}=z^{\alpha(1)+\beta(j)}\right\}
$$

is therefore a linearly independent subset of basis monomials in $\mathbb{C}[V]_{\leq \tau}$.

Remark 7.4. When $k>1$, note that $z^{\alpha(k)} \mathbf{v}_{j}$ is not in the span of $\mathcal{W}_{1}$. If it were, then all its monomials would be in $\mathcal{W}_{1}$, and, irrespective of how one orders the remaining monomials that are not in $\mathcal{W}_{1}$, the change of basis matrix on $\mathbb{C}[V]_{\leq \tau}$ from $\mathcal{C}$ to the monomial basis would not have full rank. This contradicts the fact that a change of basis matrix must be invertible.

Now, write

$$
\left[\begin{array}{c}
\mathbf{W}_{0} \\
\hline z^{\alpha(1)} \mathbf{v}_{1} \\
\vdots \\
z^{\alpha(1)} \mathbf{v}_{d} \\
\hline \operatorname{rest~of~} \mathcal{C} \\
(\operatorname{deg} \leq \tau)
\end{array}\right]=\left[\begin{array}{c}
\frac{\mathbf{W}_{0}}{\sum_{\beta} A_{1 \beta} z^{\alpha(1)+\beta}} \\
\vdots \\
\frac{\sum_{\beta} A_{d \beta} z^{\alpha(1)+\beta}}{\operatorname{rest} \text { of } \mathcal{C}} \\
(\operatorname{deg} \leq \tau)
\end{array}\right]=\left[\begin{array}{c|c|c}
I & 0 & 0 \\
\hline * & A_{(1)} & * \\
\hline 0 & 0 & I
\end{array}\right]\left[\begin{array}{c}
\frac{\mathbf{W}_{0}}{z^{\alpha(1)+\beta(1)}} \\
\vdots \\
\frac{z^{\alpha(1)+\beta(d)}}{\operatorname{rest~of~} \mathcal{C}} \\
(\operatorname{deg} \leq \tau)
\end{array}\right]
$$


where the $(j, k)$-th entry in the block $A_{(1)}$ is given by $A_{j \beta}$ with $\beta=\beta(k)$. (The '*' in the blocks adjacent to $A_{(1)}$ also consist of entries of the form $A_{j \beta}$ but do not enter into subsequent calculations.) Clearly $c \leq \operatorname{det} A_{(1)} \leq C$ as in (7.1).

Let us write this more compactly as

$$
\left[\begin{array}{l}
\mathbf{W}_{0} \\
\hline \text { rest } \\
\text { of } \mathcal{C}
\end{array}\right]=\left[\begin{array}{c|c|c}
I & 0 & 0 \\
\hline * & A_{(1)} & * \\
\hline 0 & 0 & I
\end{array}\right]\left[\begin{array}{l}
\mathbf{W}_{1} \\
\hline \text { rest } \\
\text { of } \mathcal{C}
\end{array}\right] .
$$

The ordering of the remaining monomials is done by repeating the same process as above with the polynomials $z^{\alpha(2)}, z^{\alpha(3)}, \ldots$, in turn, to form $\mathcal{W}_{2}, \mathcal{W}_{3}, \ldots$, etc. Assuming that $\mathcal{W}_{\nu-1}$ has already been constructed, consider the polynomials $\left\{z^{\alpha(\nu)} \mathbf{v}_{j}\right\}_{j=1}^{d}$. They are linearly independent, and by similar reasoning as in Remark 7.4, none of them are in the span of $\mathcal{W}_{\nu-1}$. Hence they yield $d$ additional basis monomials which, adjoined to $\mathcal{W}_{\nu-1}$, form the set $\mathcal{W}_{\nu}$. We also have an equation of the form

$$
\left[\begin{array}{c}
\mathbf{W}_{\nu-1} \\
\hline \text { rest } \\
\text { of } \mathcal{C}
\end{array}\right]=\left[\begin{array}{c|c|c}
I & 0 & 0 \\
\hline * & A_{(\nu)} & * \\
\hline 0 & 0 & I
\end{array}\right]\left[\begin{array}{c}
\mathbf{W}_{\nu} \\
\hline \text { rest } \\
\text { of } \mathcal{C}
\end{array}\right],
$$

with $c \leq\left|\operatorname{det} A_{(\nu)}\right| \leq C$ as in (7.1). This is the main formula needed for the proposition below.

Example 7.5. For the complexified sphere $\mathbf{V}\left(z_{1}^{2}+z_{2}^{2}+z_{3}^{2}-1\right)$ in $\mathbb{C}^{3}$, the elements of degree $\tau$ in the basis $\mathcal{C}$ are

$$
z_{1}^{\tau}, z_{1}^{\tau-1} \mathbf{v}_{1}, z_{1}^{\tau-1} \mathbf{v}_{2}, z_{1}^{\tau-2} z_{2} \mathbf{v}_{1}, z_{1}^{\tau-2} z_{2} \mathbf{v}_{2}, \ldots,
$$

where $\mathbf{v}_{1}=\frac{1}{2}\left(z_{2}+i z_{3}\right)$ and $\mathbf{v}_{2}=\frac{1}{2}\left(z_{2}-i z_{3}\right)$. Then

$$
\mathcal{W}_{0}=\left\{\ldots, z_{1}^{\tau}\right\}, \mathcal{W}_{1}=\left\{\ldots, z_{1}^{\tau}, z_{1}^{\tau-1} z_{2}, z_{1}^{\tau-1} z_{3}\right\}, \mathcal{W}_{2}=\mathcal{W}_{1} \cup\left\{z_{1}^{\tau-2} z_{2}^{2}, z_{1}^{\tau-2} z_{2} z_{3}\right\}
$$

Recall that for a positive integer $\tau \geq t, h_{m}(\tau-t)$ coincides with the number of multi-indices $\alpha$ for which $z^{\alpha} \mathbf{v}_{j}$ is an element in the basis $\mathcal{C}$ of degree $\tau$, where $j \in\{1, \ldots, d\}$. Introduce the notation

$$
b_{\tau}:=\sum_{s=t}^{\tau} h_{m}(s-t) .
$$

A straightforward calculation shows that

$$
b_{\tau} / l_{\tau} \rightarrow 0 \text { as } \tau \rightarrow \infty .
$$

Proposition 7.6. For any collection of points $\left\{\zeta_{1}, \ldots, \zeta_{m_{\tau}}\right\}$, with $\tau \geq t$, we have

$$
c^{b_{\tau}}\left|\operatorname{Van}\left(\zeta_{1}, \ldots, \zeta_{m_{\tau}}\right)\right| \leq\left|\operatorname{Van}_{\mathcal{C}}\left(\zeta_{1}, \ldots, \zeta_{m_{\tau}}\right)\right| \leq C^{b_{\tau}}\left|\operatorname{Van}\left(\zeta_{1}, \ldots, \zeta_{m_{\tau}}\right)\right|
$$

where $c, C$ are as in (7.1).

Proof. The proof is by induction on $\tau$. We concentrate on the upper inequality involving $C$, and note that the same proof works for the lower inequality. When 
$\tau=t$, we have

$$
\left[\begin{array}{c}
\begin{array}{c}
\text { monomials in }(*) \\
\text { of deg } \leq t
\end{array} \\
\hline \mathbf{v}_{1} \\
\vdots \\
\mathbf{v}_{d}
\end{array}\right]=\left[\begin{array}{c}
\frac{\mathbf{W}_{0}}{\sum_{\beta} A_{1 \beta} z^{\beta}} \\
\vdots \\
\sum_{\beta} A_{d \beta} z^{\beta}
\end{array}\right]=\left[\frac{I \mid 0}{A}\right]\left[\mathbf{W}_{1}\right]
$$

and note that in this case, $\left[\mathbf{W}_{1}\right]$ uses all monomials of degree $\leq t$. Forming Vandermonde determinants, we have

$$
\left|\operatorname{Van}_{\mathcal{C}}\left(\zeta_{1}, \ldots, \zeta_{m_{t}}\right)\right|=\left|\operatorname{det}\left[\frac{I \mid 0}{A}\right] \operatorname{Van}\left(\zeta_{1}, \ldots, \zeta_{m_{t}}\right)\right| \leq C\left|\operatorname{Van}\left(\zeta_{1}, \ldots, \zeta_{m_{t}}\right)\right|,
$$

where we apply (7.1) and the fact that the determinant in the middle term is the determinant of a $d \times d$ minor of $A$. This proves the base case.

Suppose the inequality holds when $\tau$ is replaced by $\tau-1$. For $j=0, \ldots, b_{\tau}$ let us introduce the convenient notation $\operatorname{Van}_{j}\left(\zeta_{1}, \ldots, \zeta_{m_{\tau}}\right)$ for the "intermediate" Vandermonde determinants:

$$
\operatorname{Van}_{j}\left(\zeta_{1}, \ldots, \zeta_{m_{\tau}}\right)=\operatorname{det}\left[\begin{array}{ccc}
\mathbf{W}_{j}\left(\zeta_{1}\right) & \cdots & \mathbf{W}_{j}\left(\zeta_{m_{\tau}}\right) \\
\hline z^{\alpha(j+1)} \mathbf{v}_{1}\left(\zeta_{1}\right) & \cdots & z^{\alpha(j+1)} \mathbf{v}_{1}\left(\zeta_{m_{\tau}}\right) \\
\vdots & \ddots & \vdots \\
z^{\alpha\left(b_{\tau}\right)} \mathbf{v}_{1}\left(\zeta_{1}\right) & \cdots & z^{\alpha\left(b_{\tau}\right)} \mathbf{v}_{d}\left(\zeta_{m_{\tau}}\right)
\end{array}\right] .
$$

In particular, $\left|\operatorname{Van}_{h_{m}(\tau-t)}\left(\zeta_{1}, \ldots, \zeta_{m_{\tau}}\right)\right|=\left|\operatorname{Van}\left(\zeta_{1}, \ldots, \zeta_{m_{\tau}}\right)\right|$.

Using equation (7.2),

$\left|\operatorname{Van}_{\nu-1}\left(\zeta_{1}, \ldots, \zeta_{m_{\tau}}\right)\right|=\left|\operatorname{det}\left(A_{(\nu)}\right)\right| \cdot\left|\operatorname{Van}_{\nu}\left(\zeta_{1}, \ldots, \zeta_{m_{\tau}}\right)\right| \leq C\left|\operatorname{Van}_{\nu}\left(\zeta_{1}, \ldots, \zeta_{m_{\tau}}\right)\right|$ for all $\nu=1, \ldots, b_{\tau}$, and hence by repeated application of the above,

$$
\left|\operatorname{Van}_{0}\left(\zeta_{1}, \ldots, \zeta_{m_{\tau}}\right)\right| \leq C^{h_{m}(\tau-t)}\left|\operatorname{Van}\left(\zeta_{1}, \ldots, \zeta_{m_{\tau}}\right)\right| \text {. }
$$

If we define $\kappa$ by the equation $\operatorname{Van}_{\mathcal{C}}\left(\zeta_{1}, \ldots, \zeta_{m_{\tau}-1}\right)=\kappa \operatorname{Van}\left(\zeta_{1}, \ldots, \zeta_{m_{\tau}-1}\right)$, then by Lemma 7.1

$$
\operatorname{Van}_{\mathcal{C}}\left(\zeta_{1}, \ldots, \zeta_{m_{\tau}}\right)=\kappa \operatorname{Van}_{0}\left(\zeta_{1}, \ldots, \zeta_{m_{\tau}}\right)
$$

as both determinants use the same elements $\left\{\mathbf{e}_{m_{\tau-1}+1}, \ldots, \mathbf{e}_{m_{\tau}}\right\}$ of degree $\tau$. Also, note that by the inductive hypothesis, we have $|\kappa| \leq C^{b_{\tau-1}}$.

Putting everything together,

$$
\begin{aligned}
\left|\operatorname{Van}_{\mathcal{C}}\left(\zeta_{1}, \ldots, \zeta_{m_{\tau}}\right)\right| & \leq C^{b_{\tau-1}}\left|\operatorname{Van}_{0}\left(\zeta_{1}, \ldots, \zeta_{m_{\tau}}\right)\right| \\
& \leq C^{b_{\tau-1}+h_{m}(\tau-t)}\left|\operatorname{Van}\left(\zeta_{1}, \ldots, \zeta_{m_{\tau}}\right)\right|=C^{b_{\tau}}\left|\operatorname{Van}\left(\zeta_{1}, \ldots, \zeta_{m_{\tau}}\right)\right|,
\end{aligned}
$$

and the induction is complete.

Theorem 7.2 is now an easy corollary.

Proof of Theorem 7.2, Let $K \subset V$ be a compact set. If $W_{m_{\tau}}=0$ for some $\tau$, then (by a similar argument as in Lemma 7.1) $W_{m_{s}}=V_{m_{s}}=0$ for all $s \geq \tau$, and the theorem follows.

Otherwise, suppose $W_{m_{\tau}}>0$ for all $\tau$. It follows easily from the above proposition that

$$
c^{b_{\tau}} W_{m_{\tau}} \leq V_{m_{\tau}} \leq C^{b_{\tau}} W_{m_{\tau}} .
$$


Using (17.3), we have $c^{b_{\tau} / l_{\tau}}, C^{b_{\tau} / l_{\tau}} \rightarrow 1$ as $\tau \rightarrow \infty$. Hence dividing by $W_{m_{\tau}}$ and taking $l_{\tau}$-th roots in (7.4), we have $\left(V_{m_{\tau}}\right)^{1 / l_{\tau}} /\left(W_{m_{\tau}}\right)^{1 / l_{\tau}} \rightarrow 1$ as $\tau \rightarrow \infty$. The theorem is proved.

We close the section by sketching an argument that shows how to get rid of the assumption that the products $z^{\alpha} z_{m}^{l} z^{\beta}$ and $z^{\alpha} z_{m}^{l} \mathbf{v}_{j}$ used in Theorem 7.2 are normal forms. In general, the methods of this section can be used to construct a basis $\mathcal{W}$ of linearly independent (but not necessarily normal form) monomials on the variety $V$, made up of the terms in these products. The same proofs also show that transfinite diameter defined in terms of $\operatorname{Van}_{\mathcal{W}}(\cdot)$ gives the same value as that defined in terms of $\operatorname{Van}_{\mathcal{C}}(\cdot)$.

Now all monomials in $\mathcal{W}$ are of the form

$$
z^{\alpha} z^{\beta}=z_{1}^{\alpha_{1}} \cdots z_{m}^{\alpha_{m}} z_{m+1}^{\beta_{m+1}} \cdots z_{n}^{\beta_{n}}
$$

with $|\beta| \leq t$, since $\operatorname{deg} \mathbf{v}_{i}=t$ for all $i$. Given $z^{\alpha} z^{\beta}$ as above, consider a monomial $z^{\alpha} z^{\tilde{\beta}}$ with $|\tilde{\beta}| \leq s$ for some $s \geq t$. Then for any compact set $K \subset V$ that avoids the coordinate axes in $\mathbb{C}^{n}$ s one can find constants $m$ and $M$, such that, upon evaluating these monomials at any point $\zeta \in K$,

$$
m^{s} \leq \frac{\left|z^{\alpha} z^{\tilde{\beta}}(\zeta)\right|}{\left|z^{\alpha} z^{\beta}(\zeta)\right|} \leq M^{s} .
$$

(For example, choose an $M>1$ such that $M \geq \frac{\max \{|z|: z \in K\}}{\left.\min \left\{\left|z_{i}\right|: z=\left(z_{1}, \ldots, z_{n}\right) \in K\right\}\right)}$.)

All elements of the (grevlex) monomial basis for $\mathbb{C}[V]$ have their total degree in the variables $z_{m+1}, \ldots, z_{n}$ uniformly bounded above (say by $s \geq t$ ), as a consequence of our hypotheses in Section 3 on Noether normalization. We can therefore compare these basis monomials to those in $\mathcal{W}$ using (7.5).

For an integer $\tau \geq t$ and collection of points $\left\{\zeta_{1}, \ldots, \zeta_{m_{\tau}}\right\} \subset K$, it follows that one can estimate the ratio $\frac{\left|\operatorname{Van} \mathcal{W}\left(\zeta_{1}, \ldots, \zeta_{m_{\tau}}\right)\right|}{\left|\operatorname{Van}\left(\zeta_{1}, \ldots, \zeta_{m_{\tau}}\right)\right|}$ with powers of $m$ and $M$, by repeatedly applying (7.5) to compare rows of the associated Vandermonde matrices. One can verify that the growth of these powers is strictly smaller, as a function of $\tau$, than the growth of $l_{\tau}$. Finally, a similar argument as carried out in the above proof (forming an equation similar to (17.4), taking $l_{\tau}$-th roots, etc.) shows that transfinite diameter defined in terms of $\operatorname{Van}(\cdot)$ gives the same value as that defined in terms of $\operatorname{Van} \mathcal{W}(\cdot)$.

\section{Appendix: The MONIC BASIS}

In 9, Rumely, Lau and Varley construct the sectional capacity of an algebraic variety. As in our case above, Zaharjuta's method plays an essential role. A so-called monic basis is constructed on the variety with good multiplicative properties, similar to those of the basis $\mathcal{C}$ from Definition 3.13 . Using the monic basis, Chebyshev constants are then defined in terms of normalized polynomial classes, and products of Chebyshev constants give the sectional capacity.

The monic basis of [9, §4] is defined in a very general, abstract setting. For simplicity, let $X \subseteq \mathbb{P}^{n}$ be an irreducible variety of dimension $m$ and degree $d$ over $\mathbb{C}$. As before, homogeneous coordinates in $\mathbb{P}^{n}$ are denoted by $z=\left[z_{0}: z_{1}: \cdots: z_{n}\right]$. Then $X$ gives the graded ring $\mathbb{C}[X]=\mathbb{C}[z] / \mathbf{I}(X)$. The monic basis is a vector space

\footnotetext{
${ }^{\S}$ Further analysis can be carried out at the end to remove this condition on the axes.
} 
basis of $\mathbb{C}[X]$ consisting of homogeneous elements $\eta_{\gamma} \in \mathbb{C}[X]_{s}$. Here is a brief sketch of how the monic basis is constructed:

(1) Write $X=X^{(0)} \supseteq X^{(1)} \supseteq X^{(2)} \supseteq \cdots \supseteq X^{(m-1)}$, where for $\ell=1, \ldots, m-1$ we have $X^{(\ell)}=\left\{z \in X^{(\ell-1)}: z_{\ell}=0\right\}$. We assume $X^{(\ell)}$ to be an irreducible variety of dimension $m-\ell$, and that the curve $X^{(m-1)}$ intersects $z_{0}=0$ in distinct smooth points of points of $X^{(m-1)}$; say on the set $D=\left\{q_{1}, \ldots, q_{d}\right\}$.

(2) Fix a sufficiently large positive integer $j_{0}$, for which the following holds for $j \geq j_{0}$ :

(a) For each $i=1, \ldots, d$ there exists a rational function on $X^{(m-1)}$ with a pole of order $j$ at $q_{i}$ and no other poles.

(b) The collection of rational functions on $X^{(m-1)}$ with poles of order at most $j$ on $D$ is isomorphic to the collection of homogeneous polynomials on $X^{(m-1)}$ of degree $j$.

(3) For each $i, j$ as above, choose a rational function $\eta_{i, j}$ (normalized appropriately) that satisfies part (a) of the previous step. Choose these functions so that the collection $\left\{\eta_{i, j}\right\}$ is multiplicatively finitely generated

(4) Use these rational functions to construct, for each $j$, a basis for the homogeneous polynomials of degree $j$ on $X^{(m-1)}$. (Note that these are polynomials in the variables $z_{0}, z_{m}, z_{m+1}, \ldots, z_{n}$ only.)

(5) Construct a basis for homogeneous polynomials on the spaces $X^{(m-2)}, \ldots$, $X^{(1)}, X$ in turn by inductively adjoining monomials in the remaining variables.

The properties of the monic basis and a justification of the above steps is given in $\S \S 4$ and 5 of [9]. See especially [9, Thm. 4.1].

Note in particular that the monic basis gives a basis of $\mathbb{C}[X]_{s}$ for every $s$. This differs from our setting, where $V \subseteq \mathbb{C}^{n}$ is an affine variety with coordinate ring $\mathbb{C}[V]=\mathbb{C}\left[z_{1}, \ldots, z_{n}\right] / I(V)$. The basis $\mathcal{C}$ we construct in Definition 3.13 consists of polynomials that restrict to a basis of $\mathbb{C}[V]_{\leq s}$ for every $s$. Thus our basis is compatible with a filtration, while the monic basis in 9 is compatible with a grading.

We illustrate how the two bases are related by examining the monic basis for the complexified sphere considered in Example 3.15.

Example 8.1. Let

$$
X=\left\{\left[z_{0}: z_{1}: z_{2}: z_{3}\right] \in \mathbb{P}^{3}: z_{1}^{2}+z_{2}^{2}+z_{3}^{2}=z_{0}^{2}\right\} \subseteq \mathbb{P}^{3},
$$

and $\mathbb{C}[X]=\mathbb{C}[z] /\left\langle z_{1}^{2}+z_{2}^{2}+z_{3}^{2}-z_{0}^{2}\right\rangle$. Then $X^{(1)}$ is the quadratic curve given by $z_{1}=z_{2}^{2}+z_{3}^{2}-z_{0}^{2}=0$ that intersects $z_{0}=0$ in $[0: 0: 1: \pm i]$.

For each $j=1,2, \ldots$, it is easy to see that

$$
\eta_{1, j}\left(z_{0}, z_{2}, z_{3}\right):=\left(\frac{z_{2}+i z_{3}}{2 z_{0}}\right)^{j}=\left(\frac{\mathbf{v}_{1}}{z_{0}}\right)^{j}
$$

defines a rational function on $X^{(1)}$ with a pole of order $j$ at $[0: 0: 1:-i]$ and no other poles. The function defined by

$$
\eta_{2, j}\left(z_{0}, z_{2}, z_{3}\right):=\left(\frac{z_{2}-i z_{3}}{2 z_{0}}\right)^{j}=\left(\frac{\mathbf{v}_{2}}{z_{0}}\right)^{j}
$$

This will ensure that the monic basis has good multiplicative properties, as can be seen in Example 8.1 below. 
has the same property in relation to $[0: 0: 1: i]$. The rational functions with at most poles of order $j$ at $[0: 0: 1: \pm i]$ are then spanned by

$$
\left\{1, \eta_{1,1}, \eta_{2,1}, \eta_{1,2}, \eta_{2,2}, \ldots, \eta_{1, j}, \eta_{2, j}\right\} \text {. }
$$

A multiplicative generating set is $\left\{1, \eta_{1,1}, \eta_{2,1}\right\}$.

Clearing denominators (i.e., multiplying by $z_{0}^{j}$ ) gives the corresponding basis of homogeneous polynomials of degree $j$ on $X^{(1)}$. For example, when $j=2$ we obtain the polynomials

$$
z_{0}^{2}, z_{0} \mathbf{v}_{1}, z_{0} \mathbf{v}_{2}, \mathbf{v}_{1}^{2}, \mathbf{v}_{2}^{2}
$$

To get the basis for the variety $X$, we adjoin powers of $z_{1}$ to basis elements for $X^{(1)}$ using the decomposition $\mathbb{C}[X]_{j}=z_{1} \mathbb{C}[X]_{j-1} \oplus \mathbb{C}\left[X^{(1)}\right]_{j}$. When $j=2$, for example, we compute that

$$
\begin{aligned}
\mathbb{C}[X]_{2} & =z_{1} \mathbb{C}[X]_{1} \oplus \mathbb{C}\left[X^{(1)}\right]_{2} \\
& =z_{1}\left(z_{1} \mathbb{C}[X]_{0} \oplus \mathbb{C}\left[X^{(1)}\right]_{1}\right) \oplus \mathbb{C}\left[X^{(1)}\right]_{2} \\
& =z_{1}^{2} \mathbb{C}[X]_{0} \oplus z_{1} \mathbb{C}\left[X^{(1)}\right]_{1} \oplus \mathbb{C}\left[X^{(1)}\right]_{2} \\
& =z_{1}^{2} \operatorname{span}\{1\} \oplus z_{1} \operatorname{span}\left\{z_{0}, \mathbf{v}_{1}, \mathbf{v}_{2}\right\} \oplus \operatorname{span}\left\{z_{0}^{2}, z_{0} \mathbf{v}_{1}, z_{0} \mathbf{v}_{2}, \mathbf{v}_{1}^{2}, \mathbf{v}_{2}^{2}\right\} \\
& =\operatorname{span}\left\{z_{0}^{2}, z_{0} z_{1}, z_{1}^{2}, z_{0} \mathbf{v}_{1}, z_{1} \mathbf{v}_{1}, \mathbf{v}_{1}^{2}, z_{0} \mathbf{v}_{2}, z_{1} \mathbf{v}_{2}, \mathbf{v}_{2}^{2}\right\} .
\end{aligned}
$$

The last line gives the monic basis for $j=2$, where the basis elements are listed according to the ordering used in 9 .

For arbitrary $j$, monic basis elements $\mathbb{C}[X]_{j}$ are either monomials in $z_{0}$ and $z_{1}$ of degree $j$, or are homogeneous polynomials of the form $z_{0}^{\alpha_{0}} z_{1}^{\alpha_{1}} \mathbf{v}_{i}^{\alpha_{2}}$ with $\alpha_{0}+\alpha_{1}+\alpha_{2}=$ $j$. Monomials in $z_{0}, z_{1}$ are listed first in lexicographic order (with $z_{0}$ preceding $z_{1}$ ), followed by elements of the form $z_{0}^{\alpha_{0}} z_{1}^{\alpha_{1}} \mathbf{v}_{i}^{\alpha_{2}}$. The latter are listed in increasing order on $i$, then lexicographically by $\alpha=\left(\alpha_{0}, \alpha_{1}, \alpha_{2}\right) \in \mathbb{Z}_{\geq 0}^{3}$. This completes the construction of the monic basis for $X$.

The monic basis constructed in Example 8.1 involves arbitrarily large powers of $\mathbf{v}_{1}$ and $\mathbf{v}_{2}$. This is related to the multiplicative properties of the monic basis described in [9, Thm. 4.1].

It is interesting to compare the monic basis of Example 8.1 to the basis constructed in Example 3.15. There, we worked with $V=\mathbf{V}\left(z_{1}^{2}+z_{2}^{2}+z_{3}^{2}-1\right) \subseteq \mathbb{C}^{3}$. Since the Zariski closure of $V$ is $\bar{V}=X=\mathbf{V}\left(z_{1}^{2}+z_{2}^{2}+z_{3}^{2}-z_{0}^{2}\right) \subseteq \mathbb{P}^{3}$, homogenization with respect to $z_{0}$ induces an isomorphism

$$
\mathbb{C}[V]_{\leq j} \simeq \mathbb{C}[X]_{j}
$$

for all $j$. It follows that the basis of Example 3.15, when restricted to elements of degree $\leq j$, gives a basis of $\mathbb{C}[X]_{j}$. However, this basis differs from the monic basis in degree $j$. For example, when $j=2$, homogenizing the basis of Example 3.15] in degree $\leq 2$ gives the homogeneous polynomials

$$
z_{0}^{2}, z_{0} z_{1}, z_{0} \mathbf{v}_{1}, z_{0} \mathbf{v}_{2}, z_{1}^{2}, z_{1} \mathbf{v}_{1}, z_{1} \mathbf{v}_{2}, z_{2} \mathbf{v}_{1}, z_{2} \mathbf{v}_{2} .
$$

Comparing this to the last line of (8.1), we see that in degree 2, the monic basis uses $\mathbf{v}_{1}^{2}$ and $\mathbf{v}_{2}^{2}$, while our basis uses $z_{1} \mathbf{v}_{1}$ and $z_{1} \mathbf{v}_{2}$. These are related by

$$
\mathbf{v}_{1}^{2}=z_{1} \mathbf{v}_{1}+\frac{1}{4} z_{1}^{2}-\frac{1}{4} z_{0}^{2}, \quad \mathbf{v}_{2}^{2}=z_{1} \mathbf{v}_{2}+\frac{1}{4} z_{1}^{2}-\frac{1}{4} z_{0}^{2} .
$$

At the conceptual level, the basis $\mathcal{C}$ constructed in Definition 3.13 focuses on the module properties of the basis, as highlighted in Theorem 3.12. In contrast, the 
monic basis constructed in 9] focuses on the multiplicative properties of the basis. In our treatment, the multiplicative properties of $\mathcal{C}$ follow from Lemma 3.7. Our construction is more direct (we avoid the inductive approach needed in [9]) but less general than that of 9 .

\section{REFERENCES}

[1] W. Baleikorocau and S. Ma'u. Chebyshev constants, transfinite diameter and computation on algebraic curves. Comput. Methods Funct. Theory, 15(2):291-322, 2015.

[2] R. Berman and S. Boucksom. Growth of balls of holomorphic sections and energy at equilibrium. Invent. Math., 181:337-394, 2010

[3] T. Bloom and N. Levenberg. Weighted pluripotential theory in $\mathbb{C}^{n}$. Amer. J. Math., 125(1):57$103,2003$.

[4] T. Bloom and N. Levenberg. Transfinite diameter notions in $\mathbb{C}^{n}$ and integrals of Vandermonde determinants. Ark. Mat., 48(1):17-40, 2010.

[5] D. Cox, J. Little, and D. O'Shea. Ideals, Varieties, and Algorithms. Springer-Verlag, New York, 2nd edition, 1997.

[6] R. Hartshorne. Algebraic Geometry. Springer-Verlag, 1977.

[7] M. Jedrzejowski. The homogeneous transfinite diameter of a compact subset of $\mathbb{C}^{n}$. Ann. Polon. Math., 55:191-205, 1991.

[8] C. F. Lau and R. Rumely. Arithmetic capacities on $\mathbb{P}^{n}$. Math. Z., 215:533-560, 1994.

[9] C. F. Lau, R. Rumely, and R. Varley. Existence of the sectional capacity. Mem. Amer. Math. Soc., 145(690), 2000.

[10] G. Martin Greuel and G. Pfister. A Singular Introduction to Commutative Algebra. Springer, Berlin, 2002.

[11] V. Zaharjuta. Transfinite diameter, Chebyshev constants, and capacity for compacta in $\mathbb{C}^{n}$. Math. USSR Sbornik, 25(3):350-364, 1975.

Department of Mathematics \& Statistics, Amherst College, Amherst, MA 01002, USA

E-mail address: dacox@amherst.edu

Department of Mathematics, University of Auckland, Auckland, NZ

E-mail address: s.mau@auckland.ac.nz 